\title{
$\frac{27}{2}+14-19+7$. \\ $3+$ t. \\ Lawrence Livermore Laboratory
}

UCID- 18168

CALCULATION OF LAMINAR INCOMPRESSIBLE FLUID FLOW AND HEAT

TRANSFER DURING SPHERICAL ANNULIJS FILLING

Dean B. Tuft

April 1979
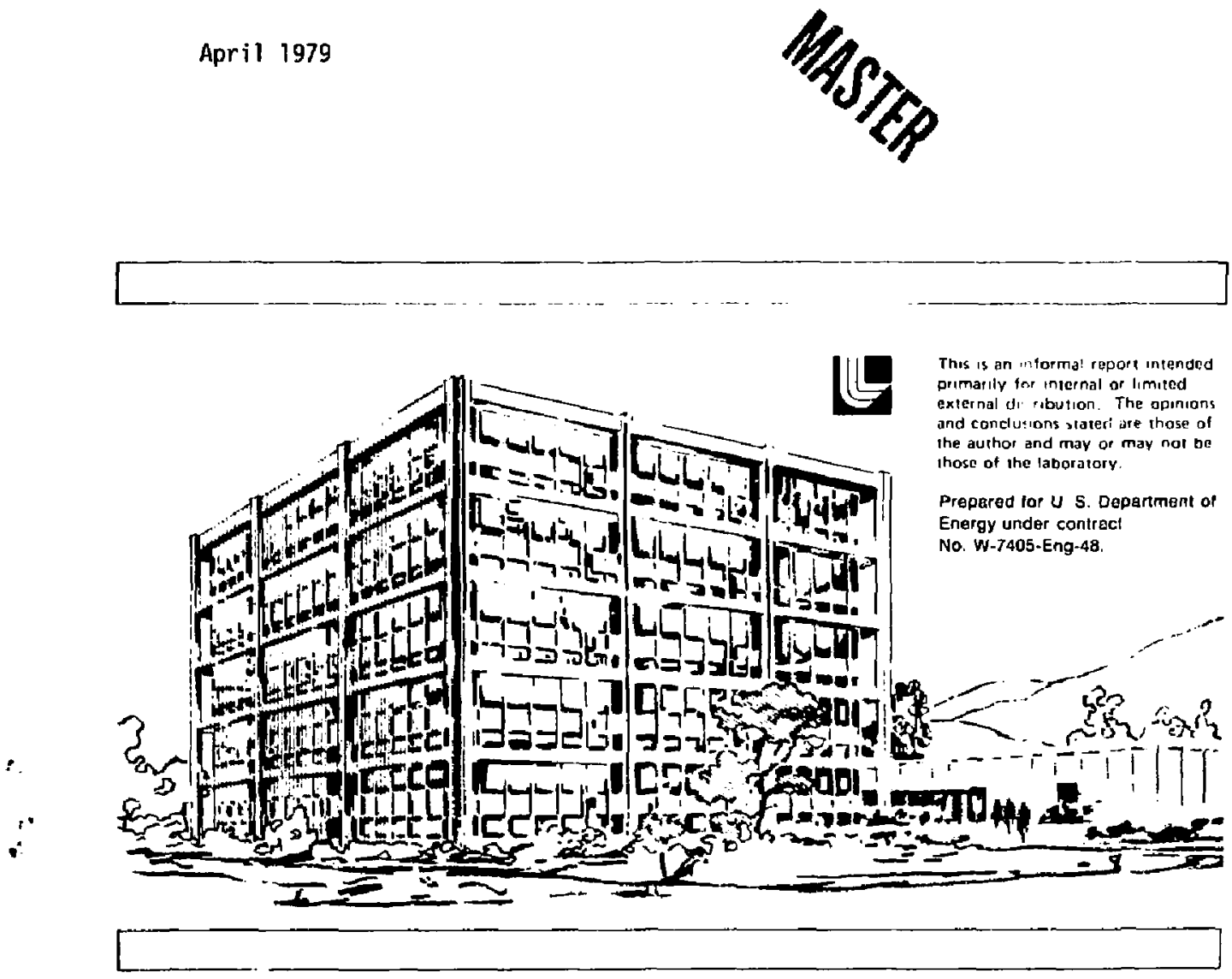


\section{CALCULATION OF LAMINAR INCOMPRESSIBLE FLUID FLOW AND HEAT TRANSFER DURING SPHERICAL ANNULUS FILLING}




\section{TABLE OF CONTENTS}

Page

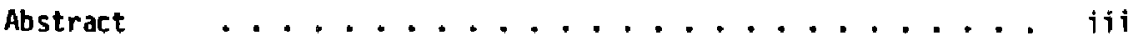

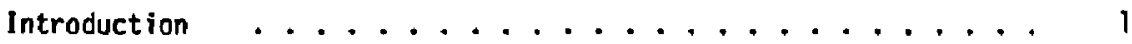

Governing Equations ................. 6

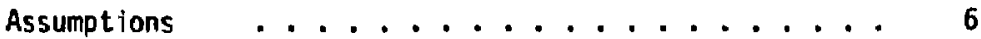

Vector Equations .............. 8

Differential Equations ................... 10

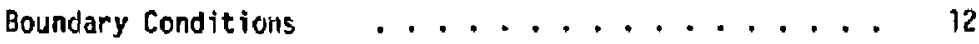

Numerical Solution .................. 74

Finite-Cifference Equations .......... 14

Numerical Solution Procedure ......... 18

Fin ite-Difference Boundary Conditions ....... 24

Free Surface Treatment ........... 26

Computational Results ................... 27

Sumary and Conclusions ................ 38

References ................... 40

Appendix $\bar{A} \quad$ Nonstandard Terms for Finite-Difference

Equations .............. 42

Appendix B Numerical Stability Conditians . . . . . . 45

Appendix $C$ Conservation of Vorticity by Pressure-

Velocity Iterations, the Poisson Equation

for Pressure, and the MAC Corrective Term . . 49

Appendix D Details of Free Suriace Treatment . . . . . 54

Appendix E The Computer Code SAFFA . . . . . . . 78

Appendix $f$ Nomenclature . . . . ....... 80 


\section{ABSTRACT}

In this paper, a method of computing laminar incompressible fluid-flow and neat transfer during the filling of a spherical annulus is presented. Transient fluid temperatures and heat flux rates in the spherical annulus are calculated for an insulated outer sphere and a constant temperature inner sphere with heated water filling the annulus from the bottom.

To achieve a solution, laminar axidlly symmetric flow is assumed and the Marker-and-Cell (MAC) free surface computational method is applied to this problem in spherical coordinates. Changes in the standard MAC treatment are incorporated and special methods for handling the free surface are introduced. A variable mesh is used to improve resolution near the inner sphere where temperature and velociiy gradients are steep and the governing equations are derived for variable fluid properties to allow an eddy viscosity turbutence model to be applied Tater.

Calculations of velocity, temperature, and inner sphere heat flux in a spherical annulus of $139.7 \mathrm{~mm}$ inner radius, and $168.3 \mathrm{~mm}$ outer radius within an inlet tube diameter of $38.1 \mathrm{~mm}$ are presented. The inner sphere is held at $0^{\circ} \mathrm{C}$ and the inlet water temperature is $50^{\circ} \mathrm{C}$. Laminar results corresponding to an annulus fill time of 20 seconds are presented and the major features of the flow are discussed.

Computed separation point location is compared to experimental results from the literature. Differences in computed and experimental separation point location are shown to be a result of turbulence in the 
experiments which was not accounted for in the calculations. Calculated flow patterns are in qualitative agreement with steady flow experimental observations in the literature.

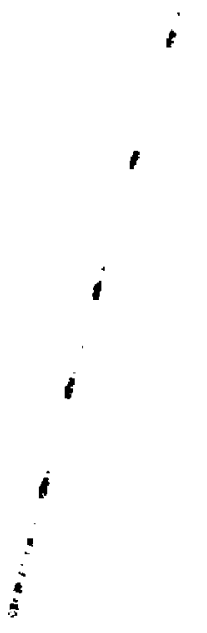




\section{INTRODUCTION}

The spherical annulus is defined as the region between concentric spheres. This geometry is advantageous in certain heat transfer applications because it provides the maxinum volume per unit surface area. Heat transfer applications for steady state flow between two concentric spheres include cryogenic storage systems and guard heating systems in which a material inside the inner sphere is maintained at constant temperature by fluid in the annulus. A similar steady flow application is the cooling of gyroscopic gimbals by fluid flow in a surrounding spherical annulus. In addition, steady flow spherical shell heat exchangers are used in homogeneous nuclear reactors to cool the spherical fue? elements.

To date, the majority of the spherical annulus flow research has been applied to the study of steady forced or naturat convection flow in a spherical shell heat exchanger. One of the first analytical treatments of fluid flow in a spherical shell was by Cobble (1). Cobble assuned a tangential velocity distribution and then calculated heat transfer based on the energy equation. Bird, Stewart and Lightfoot (2), presented the solution to isothermal creeping flow in a spherical annulus. Ward (3) provided a flow visualization study of isothermal flow in a spherical annuius between 60 and 120 degrees downstream of the entrance. He showed that the velocity profile cannot be predicted analytically by neglecting the radial velocity component. In addition, Ward stated that if the radial velocity component were left in the governing equations a simple analytical solution would not be possible and numerical methods would be 
required. Rundelt and Rundell et. a). $(4,5)$ measured the temperature profiles between inner and outer spheres and the bulk heat transfer coefficient for steady flow in a spherical shell heat exchanger. They obtained a heat transfer correlation for two sets of sphere sizes which was later extended by Cox et. al. (6) to include other sphere sizes. Bozeman and Bozeman et. al. $(7,8)$ added to the isothermal flow visualization work of Ward by focusing on the entrance region. They state that the most significant heat transfer occurs upstream of separation in the region near the inlet where the flow impinges on the inner sphere and jets off tangentially.

Rundell observed a flow rate independant separation point located between 45 and 50 degrees downstream of the entrance. Bozeman added that upstream of the separation point the flow is characterized by a high velocity jet of fluid near the inner sphere with a relatively low velocity return flow near the outer wall. Downstream of separation, the main flow moves directly to the outer sphere creating a low velocity return flow near the inner sphere. The high velocity jet near the inner sphere upstream of the separation, point makes this an area of significant heat transfer. Beyond the separation point, the fluid is moving slowly near the inner sphere and as a result a lower heat transfer rate is expected in that region.

The problem of laminar natural convection flow in a closed spher $i-$ cal annulus was solved numerically by Brown (9). Brown solved the vorticity and temperature equations in spherical coordinates by an explicit finite-difference technique coupled to an iterative solution of the vorticity-stream function relation. The majority of the calculations 
were for air while some calculations for water and mercury were included. Astill (10) applied a boundary-layer order of magnitude analysis to the dimensionless forced convection equations in spherical coordinates to reduce them to a set of parabolic differential equations. He simplified the inlet and outlet flow conditions by assuming a uniform velocity profile across the annulus at specified inlet and out let angles. The solution was olstained by a finite-difference method that marches forward in the az imuthal angle.

In this study, we are interested in calculating water temperatures and heat flux rates during hot water filling of a spherical annulus in which the inner sphere is isothermal and the outer sphere is insulated. Laminar, incompressible, axially symetric flow is assumed and the governing equations of mass, momentum, and energy conservation are solved in spherical coordinates. A variable mesh is used to improve resolution near the inner sphere where velocity and temperature gradients are large. Variable fluid properties are assumed so that an eddy viscosity type turbulence model may be added later.

The momentum equations are solved explicitly for the radial and azimuthal velocity components and these velocities are iteratively adjusted along with pressure until mass conservation is satisfied. Temperatures are obtained explicitiy from the energy conservation equation which is coupled to the momentum equation by the fluid velocity. The Marker-and-Cell (MAC) computational technique (11) is modifed to handle the free surface aspects of this problem. A sketch illustrating the basic flaw problem is shown in Fig. 1. 


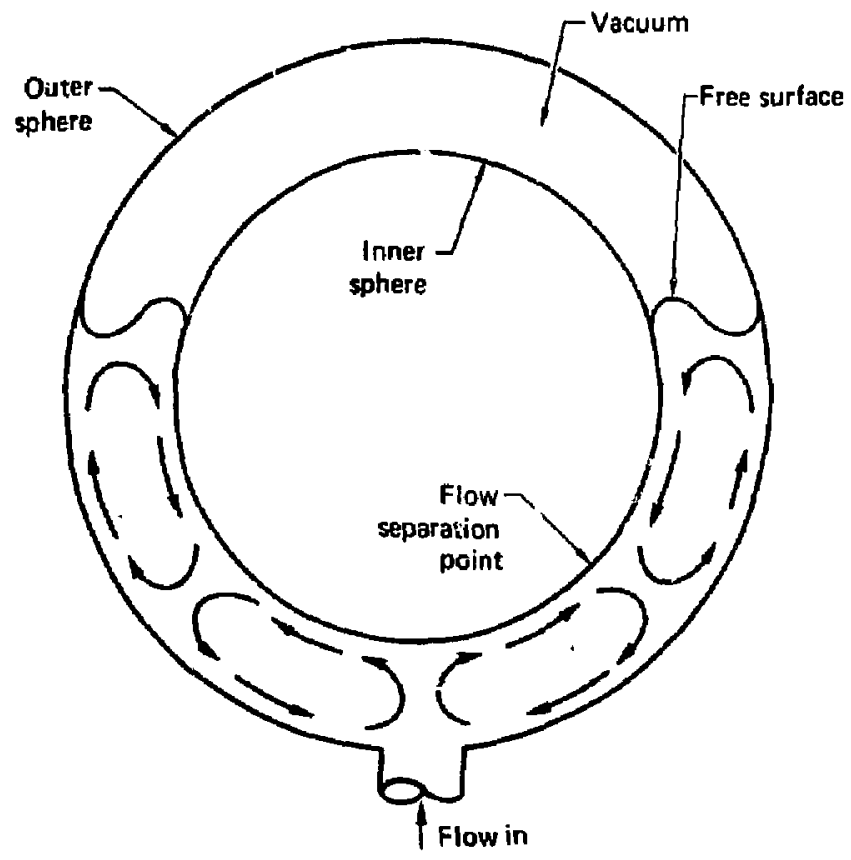

Figure 1. Basic configuration for spherical annutus filling. 
To the best of our know? edge, this is the first study of the transient filling of a spherical annulus by either experimental or computational methods. However, we have an idea of the nature of the transient flow problem from the steady flow visualization work reported in the 1 iterature $(3,5,8)$. As the spherical annulus fills, flow in regions away from the free surface soon estabtishes steady patterns. Flow in the inlet region, where the majority of the heat transfer occurs, becomes steady quite early in the filling process. As a result, a similar fiow pattern and separation point location are expected in this resion for the transient filling problem as was observed for steady flow in a full annulus. Results from Refs. (3), and (5) on separation point will be compared to our computational solution. 


\section{GOVERNING EQUATIONS}

The equations governing heat and mass transfer during the filling of a spherical annulus are the conservation equations of mass, radial momentum, azimutha 1 momentum and energy. In the solution of these equations, we use the primitive variables of pressure, velocity, and temperature and solve the equations in the spherical $r$. o plane shown in Fig. 2. The azimuthal coordinate 0 , is measured from the lower lle and the radius is measured from the comon center. The sphere radaii are denated as $R_{1}$ for the inner sphere and $R_{2}$ for the outer sphere.

\section{ASSIMMTIONS}

For all equations, we assume laminar incompressible axially symmetric flow. Hard (3) and Rundell (4) have shown experimentally that axially symmetric flow is a valid assumption for the spherical annulus with uniform inlet flow. Nithough the flow in the spherical annulus is turbulent for most flow rates $(3,5,8)$, laminar flow is assumed for simplicity and as a starting point for the analysis. The computational techrique is essentially the same for variable property laminar flow as for turbulent flow with an effective eddy viscosity and therinal diffusivity. The equations are derived for variable thermal diffusivity and variable viscosity to allow a turbulence model to be incorporated later.

In the momentum equaticns, surface tension and buoyancy forces are neglected and in the energy equation, compression work and viscous dissipation are neglected. In addition, zero free surface heat loss and no phase chariges are assumed. 


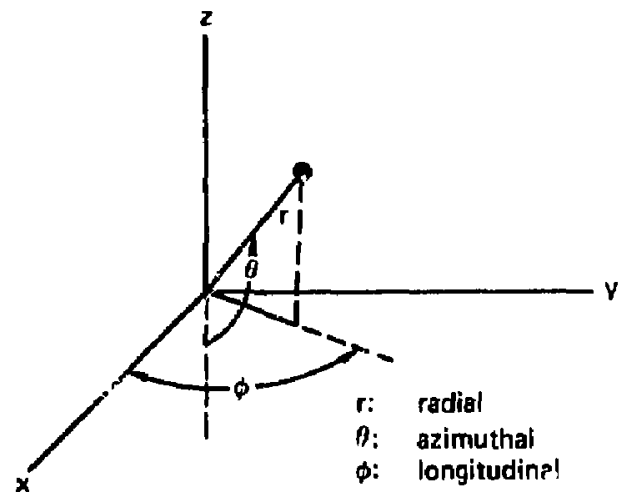

(a) Spherical coordinate system

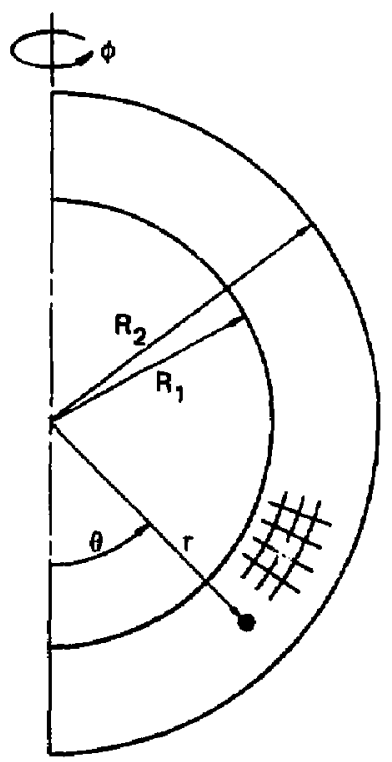

(b) Spherical annulus coorainates

Figure 2. Spherical coordinates -- Application to the spherical annulus. $-7-$ 
VECTOR EQUATIONS

The vector equation expressing conservation of momentum for transient laminar incompressible fluid flow is

$$
\frac{\partial \vec{U}}{\partial t}=-\vec{U}(\vec{U} \cdot \vec{\nabla})-\frac{\overrightarrow{\nabla P}}{\rho}-\vec{\nabla} \times[\nu(\vec{\nabla} \times \vec{U})]+\dot{g}
$$

The velocity vector is defined as $\vec{U}=\hat{i}+\hat{j} v$, where $\hat{i}$ is the unit vertor in the $r$ direction, $\hat{j}$ is the unit vector in the $\theta$ direction, and $u$ and $v$ are the velocity components. The kinematic viscosity is represented by $v$, the fluid density by $\rho$ and $v$ represents the spherical gradient vector operator. Pressure is denoted by $P, t$ ime by $t$, and the gravity vector is defined by $\vec{g}=\hat{i} g_{\mu}+\hat{j} g_{g}$.

The momentum equation represents a balance of fluid momentum. Each individual term expresses a separate source of momentum change in a fluid element. The term on the left represents the time rate of change of monentum. The first term on the right expresses the convection of momentum by fluid motion and the second term represents momentum changes due to normal pressure forces. The next term is the mathematical representation of diffusion of momentum by viscous forces and the last term on the right expresses momentum production by body forces due to gravity.

Along with the momentum equation, a statement of mass conservation is required. The vector form of the continuity equation for incompressible flow is

$$
(\vec{\nabla} \cdot \vec{U})=0
$$


The continuity equation states that mass must be conserved and acts as an incompressibility condition for the problem. The momentum equation is coupled to the continuity equation by the velocity.

For numerical solution, equation 2 is used to modify the convective terms in equation 1 via the vector identity

$$
\vec{U}(\vec{U} \cdot \vec{\nabla})=\ddot{\nabla} \cdot(\vec{U} \vec{U})-\vec{U}(\vec{\nabla} \cdot \vec{U})
$$

Using equations 2 and 3 , equation 1 written in conservative form becomes

$$
\frac{\partial \vec{U}}{\partial t}=-\vec{\nabla} \cdot(\vec{U} \vec{U})-\frac{\vec{\nabla} P}{p}-\vec{\nabla} \times[\nu(\vec{\nabla} \times \vec{U})]+\vec{g}
$$

Equation 4 is the governing vector monentum equation for this study. By integrating each term in equation 4 over a control volume and by using the divergence theorem to convert volume integrals to surface integrals, it can be shown that this equation equates the flux of momentum through the control surface to the time rate of clange of momentum in the control volume plus the change of momentum due to body forces. By using this form of the equation, we are directly equating changes of momentum within the control volume to fluxes of momentum through the control surface. In this form, the equation is a direct statement of conservation of momentum and avoids nonconservative errors that are inherent in other forms of the equation. Roache, (13) states that the conservative form when passed on to the finite-difference equations is generally more accurate than nonconservative forms. In this solution, finite-difference techniques that preserve the conservative property of the equations are used. 
The energy conservation equation can also be written in conservative form by using equation 2. The conservative form of the energy equation is

$$
\frac{\partial T}{\partial t}=-\vec{\nabla} \cdot(\vec{U} T)+\vec{\nabla} \cdot(a \vec{\nabla} T)
$$

In the above equation, "a" represents the thermal diffusivity. The left hand side represents the time rate of change of lccal energy. The first term on the right side of the equation represents the convection of energy by fluid motion and is the term through which the energy equation is coupled to the monentum equation. The last term on the right is the heat conduction term.

DIFFERENTIAL EQUATIONS

In this axisymetric flow problem, there are two differential momentum equations, one for each independent coordinate axis . The differential momentum equations are

\section{Radial Mamentum:}

$$
\begin{aligned}
& \frac{\partial u}{\partial t}+\frac{1}{r^{2}} \frac{\partial}{\partial r}\left(r^{2} u^{2}\right)+\frac{1}{r \sin \theta} \frac{\partial}{\partial \theta}(u v \sin \theta)-\frac{v^{2}}{r}=-\frac{1}{\rho} \frac{\partial P}{\partial r} \\
& +\frac{1}{r^{2} \sin \theta}\left\{\frac{\partial}{\partial \theta}\left(\nu \sin \theta \frac{\partial u}{\partial \theta}\right)-\frac{\partial}{\partial \theta}\left(\nu \sin \theta \frac{\partial}{\partial r}(r v)\right)\right)+g_{r} .
\end{aligned}
$$


Azimuthal Momentum:

$$
\begin{gathered}
\frac{\partial v}{\partial t}+\frac{1}{r^{2}} \frac{\partial}{\partial r}\left(r^{2} u v\right)+\frac{1}{r \sin \theta} \frac{\partial}{\partial \theta}\left(v^{2} \sin \theta\right)+\frac{u v}{r}=-\frac{1}{\rho r} \frac{\partial P}{\partial \theta} \\
+\frac{1}{r}\left\{\frac{\partial}{\partial r}\left(\nu \frac{\partial}{\partial r}(r v)\right)-\frac{\partial}{\partial r}\left(\nu \frac{\partial u}{\partial \theta}\right)\right\}+g_{\theta} .
\end{gathered}
$$

The term $\left(v^{2} / r\right)$ in the radial equation, and $(u v / r)$ in the azimuthal equation do not appear in rectangular coordinates. These are the centrifugal and Coriolis terins respectively, and are a result of the curvature of the spherical coordinate system.

The differential form of the continuity equation is

$$
\frac{1}{r^{2}} \frac{\partial}{\partial r}\left(r^{2} u\right)+\frac{1}{r} \frac{1}{\sin \theta} \frac{\partial}{\partial \theta}(v \sin \theta)=0
$$

and the differential form of the energy equation is

$$
\begin{aligned}
\frac{\partial T}{\partial t}+ & \frac{1}{r^{2}} \frac{\partial}{\partial r}\left(r^{2} u T\right)+\frac{1}{r \sin \theta} \frac{\partial}{\partial \theta}(v T \sin \theta)= \\
& \frac{1}{r^{2}} \frac{\partial}{\partial r}\left(a r^{2} \frac{\partial T}{\partial r}\right)+\frac{1}{r^{2} \sin \theta} \frac{\partial}{\partial \theta}\left(\operatorname{asin} \theta \frac{\partial T}{\partial \theta}\right) .
\end{aligned}
$$

Equations $6,7,8$, and 9 , are the differential equations governing this problem. They are four differential equations written in terms of four independent unknowns. The unknowns are, radial velocity $u$, azimuthal 
velocity $v$, pressure $P$, and temperature $T$. The momentum and energy equations are coupled, nonlinear, parabolic jartial differential equations. An explicit finite-difference time marching scheme and an iterative technique will be used to solve the momentum and continuicy equations for the two velocity components and the pressure. Temperatures will then be found using the new velocities and an explicit finite-difference solution of the energy equation.

\section{BOUNDARY CONDITIONS}

The governing equations require boundary conditions on velocity, pressice and temperature at each physical boundary $\left(r=R_{1}\right.$, and $r=$ $\left.R_{2}\right)$ along symmetry lines at the sphere poles $(\theta=0$ and $\theta=\pi)$. Both normal and tangential velocity components are specified at sphere walls. The nornal relocity is zero at a wall except at the inlet where it is constant. The tangential velocity at a wall is zero for a no-slip condition or calculated to give zero tangential shear stress for a free-slip condition. The symmetry lines at $\theta=0$ and $\varphi=\pi$ are treated as free-slip boundaries. Free surface conditions on velocity and pressure are given special attention in Appendix $D$.

Application of explicit boundary conditions on pressure at the walls is not required because a slope boundary condition is implicitly applied by the solution technique. This will be discussed in the section on numerical procedure. In addition, singularities in the equations at $\theta=0$ and $\theta=\pi$ are avoided by the placement of velocities in the finite-difference mesh.

The boundary conditions can be summarized as follows 


\section{Velocity:}
$u=u_{\text {in }}$
$0 \leqq \theta \leqq \theta_{\text {in }} \quad r=R_{2}$
$\mathbf{u}=0$
$0 \leqq \theta \leqq \pi \quad r=R_{1}, r=R_{2}$
$\frac{\partial u}{\partial \theta}=0$
$6=0, \pi \quad R_{1} \leqq r \leqq R_{2}$
$v=0$ or $\frac{\partial}{\partial r}(v / r)=0$
$0 \leqq \theta \leq \pi \quad r=R_{1}, r=R_{2}$
Temperature:
$T=T_{\text {in }}$
$0 \leq \theta \leq \theta_{\text {in }} \quad r=R_{2}$
$\frac{\partial T}{\partial r}=0$ or $T=T_{0}$
$0 \leqq \theta \leqq \pi$
$r=R_{1}, r=R_{2}$
$\frac{\partial T}{\partial \theta}=0$
$\theta=0, \pi \quad R_{1} \leqq r \leqq R_{2}$ 


\section{NUMERICAL SOLUTION}

\section{FINITE-DIFFERENCE EQUATIONS}

To achieve a numerical solution of the governing equations, we divide the flow region into finite cells and approximate derivatives by finite-differences. Mesh variable placement as in the MAC method (14) is shown in Fig. 3 and half cell notation is used to indicate cell face variables, For simplicity, constant size cells are used in this and all following figures. With variables located as shown in Fig. 3 , the conservative property of the differential equations is preserved in the finite-differenc- equations. By applying a forward-time centered-space (13) finite-difference approximation for a variable mesh to equations 6 and 7 and denoting time step by superscript $n$, we have equations 10 and 11 shown in Figs. 4 and 5 . The numerical time increment is represented by $\Delta t$ and the fluid viscosity by $v_{i, j}$, Viscosity may be calculated as a function of temperature or as an effective turbulence parameter. In this paper, constant laminar viscosity is used and turbulence modeling is left to be dane as a continuation of this study.

The form of the convection terms allows variable donar cell differencing. With $\alpha=1$ the equations are equivalent to full donor cell "upwind" differencing and with $\alpha=0$ they reduce to centered differencing. Centered differencing of convection terms is unstable and $\alpha$ must be siightly larger than the maximum of $\left|\frac{u \Delta t}{\Delta r}\right|$ or $\left|\frac{v \Delta t}{r \Delta \theta}\right|$ occurring in the mesh (15). Donor cell differencing provides a more physically realistic treatment of convective derivatives because it allows information to be 


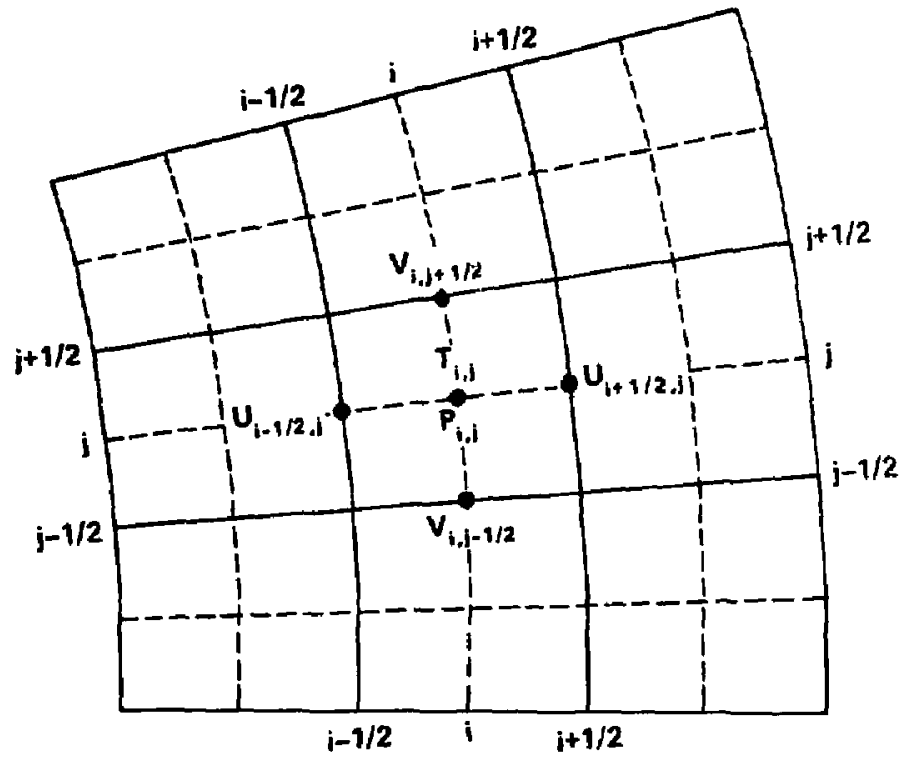

Figure 3. Location of variables in the finite-difference mesh. 


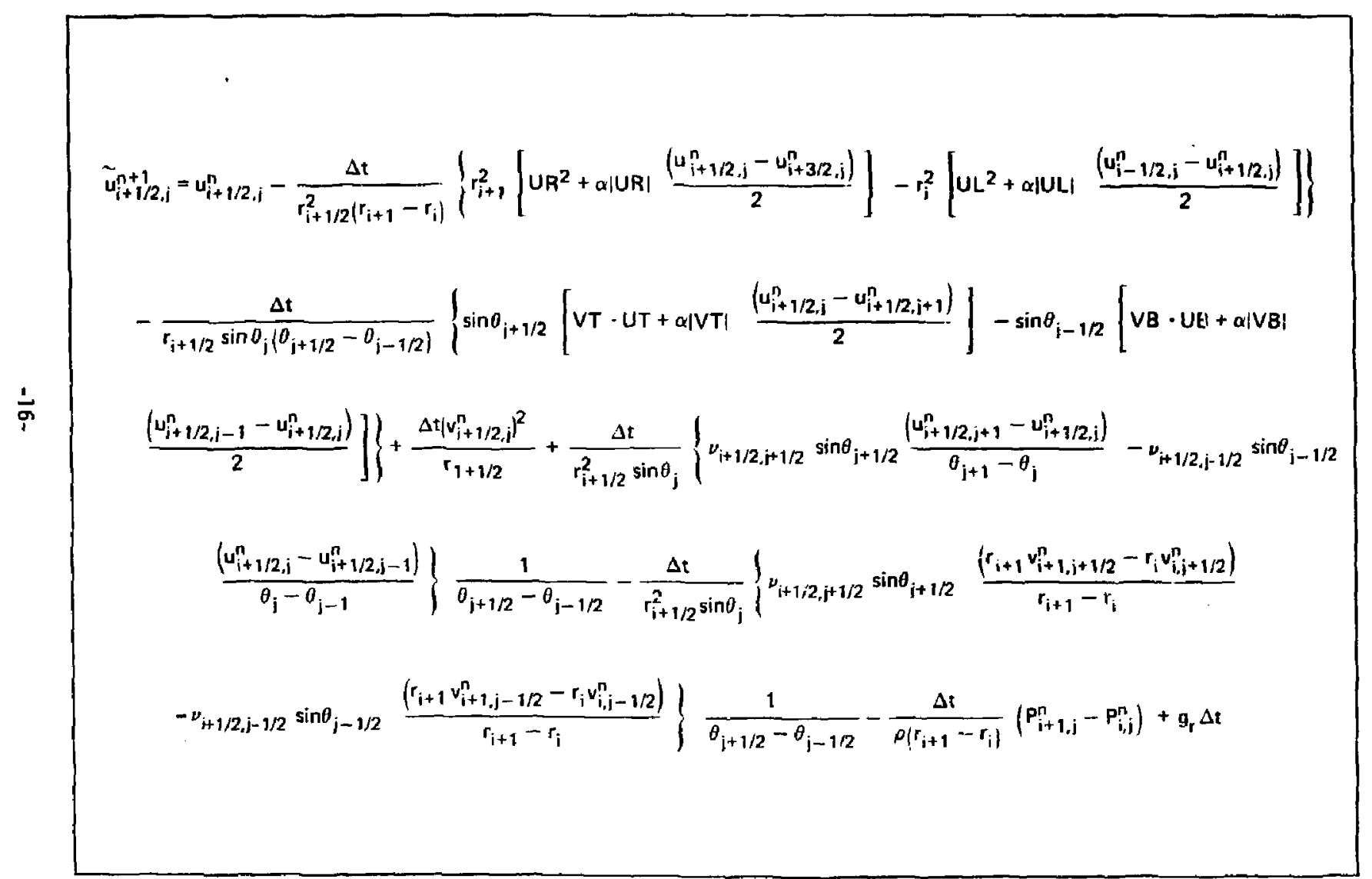

Figure 4. Finite-difference form of radial momentum equation (equation 10). 


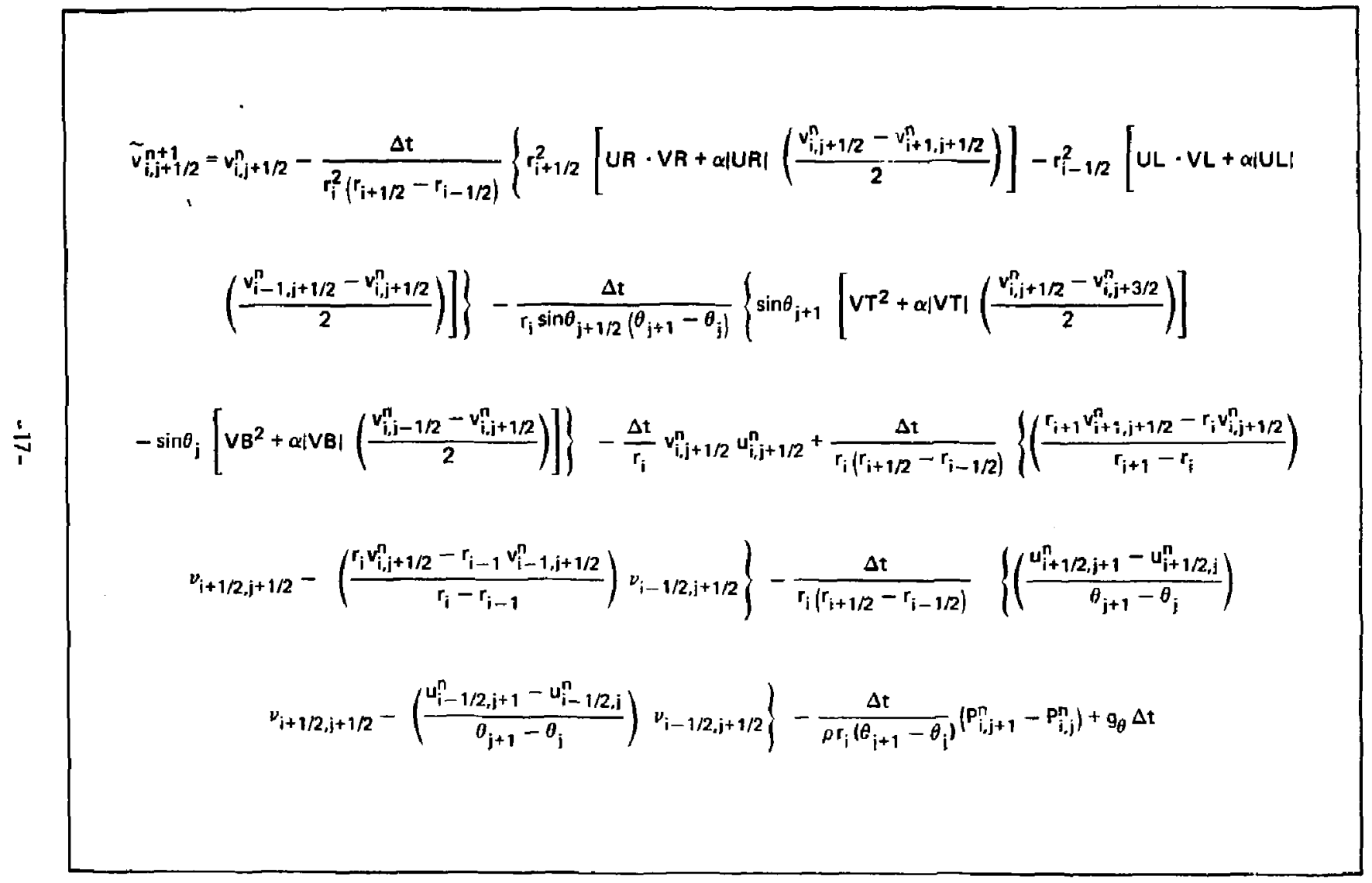

Figure 5. Finite-difference form of azimuthal momentum equation (equation 11). 
convected downstream only. Partial donor cell differencing will result in less numerical diffusion than full donor cell differencing, but care must be taken not to exceed the above limits on alpha.

By applying variable donor cell differencing to the convective terms in the energy equation and forward-time centered-space finite-differences to all other terms, we have the finite-difference energy equation shown in Fig. 6 . The term $a_{i, j}$ represents the thermal diffusivity of the fluid and is defined on cell faces.

Terms such as VT, VB, UT. UB, TT, TB, etc. in equations 10, 11 , and 12 are velocities anu temperatures that do not lie on the usual mesh point locations shown in Fig. 3. These terms are calculated by mass flow weighted averages to account for changes in cell areas and are listed in Appendix $A$ in terms of regular mesh point variables.

Spatially centered finite-differences are used to write the continuity equation in finite-difference form.

\section{Continuity Equation:}

$$
\begin{gathered}
D_{i, j}=\frac{1}{r_{i}^{2}}\left[\frac{r_{i+1 / 2}^{2} u_{i+1 / 2, j}^{n}-r_{i-1 / 2}^{2} u_{i-1 / 2, j}^{n}}{r_{i+1 / 2}-r_{i-1 / 2}}\right] \\
+\frac{1}{r_{i} \sin \theta_{j}}\left[\frac{\sin \theta_{j+j / 2} v_{i, j+1 / 2}^{n}-\sin \theta_{j-1 / 2} v_{i, j-1 / 2}^{n}}{\theta_{j+1 / 2}-\theta_{j}-1 / 2}\right]=0
\end{gathered}
$$

NUMERICAL SOLUTION PROCEDURE

The numerical solution of these finite-difference equations proceeds in three steps. The first step is the explicit solution of 


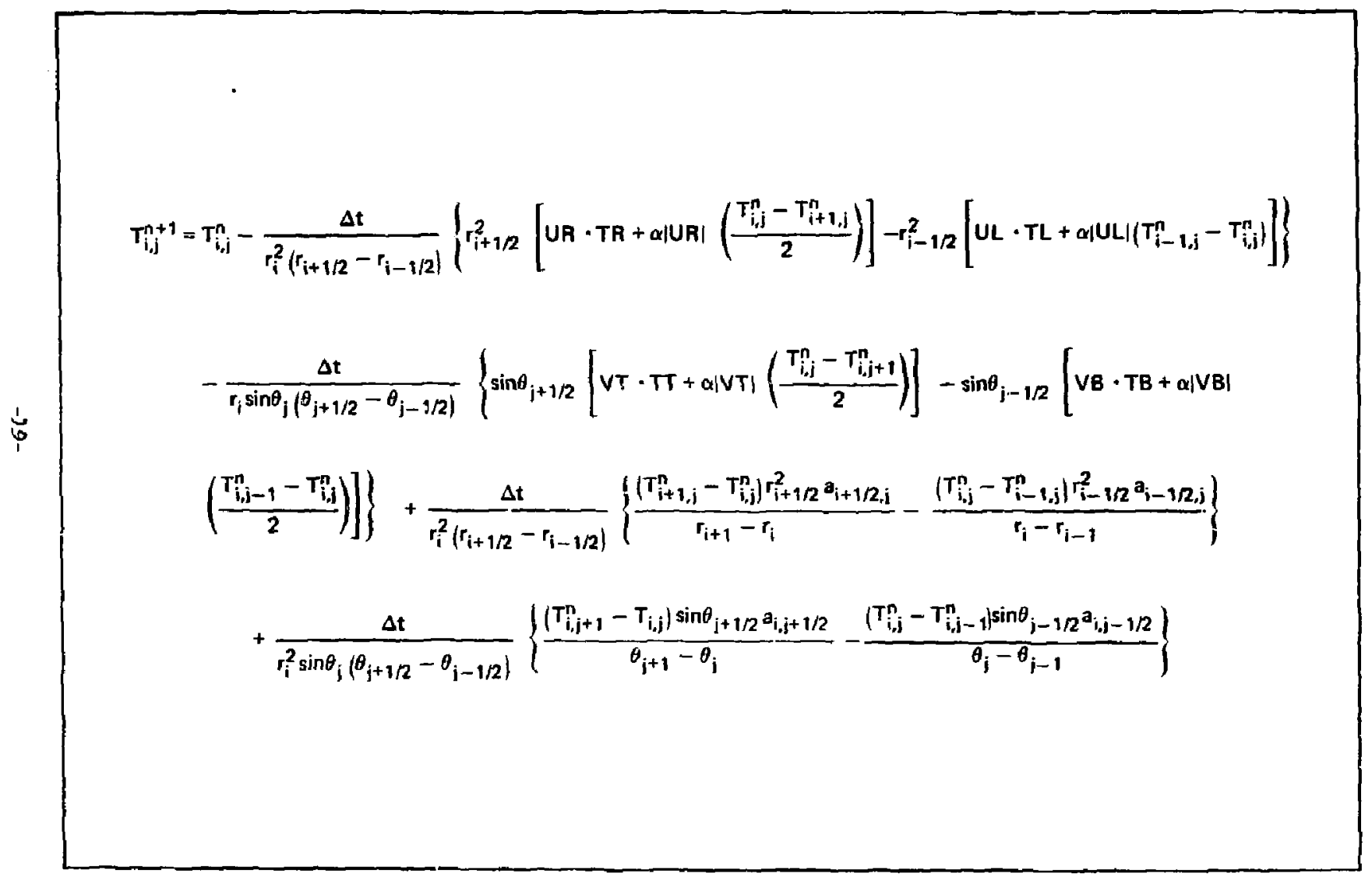

Figure 6. Finite-difference form of energy equation (equation 12). 
finite-difference equations 10 and 11 for $\tilde{u}_{i+1 / 2, j}^{n+1}$ and $\tilde{v}_{i, j+1 / 2}^{n+1}$. The tilde denotes that these ars tentative velocities which are not auite complete because they do not in genera? satisfy conservation of mass. In the second step of the solution we impose equation 13 and simultaneously iterate pressures and velocities until mass conservation is achieved. An equivalent technique was introduced by Chorin (16) and has been applied to MAC calculations by others $(17,18)$. Pressure-velocity iterations are performed in such a way as to preserve the vorticity of the original tentative velocity field. Iterations are continued until the maximum cell divergence drops belaw a specified limit. At this point the solution for velocity and pressure at the rew time step is achiever. In the third step, equation 12 is solved explicitiy for temperature using the velocity solution from step two.

To achieve the pressure-velocity iterative solution in step tro. equations 10 and 11 are written in condensed form for the velocity components on the sides of a cell.

$$
\begin{aligned}
& u_{i+1 / 2, j}^{n+1}=\tilde{u}_{i+1 / 2, j}^{n+1}+\frac{\Delta t}{\rho\left(r_{i+1}-r_{i}\right)} \Delta P_{i, j}^{l} \\
& u_{i=1 / 2, j}^{n+1}=\tilde{u}_{i-1 / 2, j}^{n+1}-\frac{\Delta t}{\rho\left(r_{j}-r_{i-1}\right)} \Delta P_{i, j}^{l} \\
& v_{i, j+1 / 2}^{n+1}=\tilde{v}_{i, j+1 / 2}^{n+1}+\frac{\Delta t}{\rho r_{i}\left(\theta_{j}+1-A_{j}\right)} \Delta P_{i, j} \\
& v_{i, j=1 / 2}^{n+1}=\tilde{v}_{i, j-1 / 2}^{n+1}-\frac{\Delta t}{\rho r_{i}\left(\theta_{j}-\theta_{j-1}\right)} \Delta P_{i, j}^{l}
\end{aligned}
$$


The tilde velocities in the above equations contain ali terms from the right hand side of equations 10 and 11 including the pressure derivatives. We have added a pressure adjustment term $\Delta \mathbf{p}^{I}$ to adjust the pressure at the $I_{\text {th }}$ iteration according to

$$
P_{i, j}^{++l}=P_{i, j}^{l}+\Delta P_{i, j}^{I} .
$$

The pressure adjustment term, $\Delta P_{i, j}^{I}$ is calculated to force the velocity field to satisfy the continuity equation locally. The equation for $\Delta P^{I}$ is found by substituting equations 14 into equation 13 and solving for $\Delta \mathrm{P}^{\mathrm{I}}$. The result is

$$
\Delta P_{i, j}^{I}=-\frac{\omega_{0}}{\Delta t \beta_{i, j}}\left[D_{i, j}\right]
$$

In the above equation, $D_{i, j}$ represents the finite-difference velocity divergence for cell $(i, j)$ given by equation 13 and $\beta_{i, j}$ is a geometry factor.

$$
\begin{aligned}
\beta_{i, j} & =\frac{1}{r_{i}^{2}}\left\{\frac{1}{\Delta r_{i}}\left(\frac{r_{i+1 / 2}^{2}}{\Delta r_{i+1 / 2}}+\frac{r_{i-1 / 2}^{2}}{\Delta r_{i-1 / 2}}\right]\right\} \\
& +\frac{1}{r_{i}^{2} \sin \theta_{j}}\left\{\frac{1}{\Delta \theta_{j}}\left[\frac{\sin \theta_{j+1 / 2}}{\Delta \theta_{j+1 / 2}}+\frac{\sin \theta_{j-1 / 2}}{\Delta \theta_{j-1 / 2}}\right]\right\},
\end{aligned}
$$

where:

$$
\begin{aligned}
& \Delta r_{i}=r_{1+1 / 2}-r_{i-1 / 2}, \Delta r_{i+1 / 2}=r_{i+1}-r_{i}, \Delta r_{i-1 / 2}=r_{1}-r_{i-1} . \\
& \Delta \theta_{j}=\theta_{j+1 / 2}-\theta_{j-1 / 2}, \Delta \theta_{j+1 / 2}=\theta_{j+1}-\theta_{j}, \Delta \theta_{j-1 / 2}=\theta_{j}-\theta_{j-1} .
\end{aligned}
$$


In equation 16, we have introduced a relaxation parameter $\omega_{0}$ to accelerate convergence (19). For stability, $\omega_{0}$ has the restriction $0<\omega_{0}<2$. We have found that $\omega_{0}=1.8$ provides the most rapid rate of convergence for our problem. Variations in $\omega_{0}$ near the free surface are discussed in Appendix 0 .

If properly calculated, $\beta_{i, j}$ will have a form for cells adjacent to walls that is different from the form for interior cells given by equation 17. Because the normal velocity component is zero at a wall the term containing $r_{j+1 / 2}^{2}$ will drop out near the outer wall and the term containing $r_{j-1 / 2}^{2}$ will vanish near the inner wall. Essentially $\beta_{i, j}$ has a different form near walls because wall cells have only three degrees of freedom (i.e., velocity components that may be iteratively adjusted) as opposed to four for interior cells. In adjusting $\beta_{i, j}$ for wall cells, we are effectively over-relaxing these cells an additionat amount to account for the reduced degree of freedom.

We begin the iteration to achieve mass conservation by calculating the cell velocity divergence from equation 13 and finding a pressure increment from equation 16 . The pressure is then incremented in each cell by equation 15 and cell velocity components are updated according to equations 14. The entire mesh is swept and the same adjustments are made for each cell. After the first iteration sweep the tilde velocities are averwritten by the new adjusted velocities and the iteration equations for the cell velocity components take on the form 


$$
\begin{aligned}
& u_{i+1 / 2, j}^{n+1} \rightarrow u_{i+1 / 2, j}^{n+1}+\frac{\Delta t}{\rho\left(r_{i+1}-r_{i}\right)} \Delta P_{j, j}^{l}, \\
& v_{i, j+1 / 2}^{n+1} \rightarrow v_{i, j+1 / 2}^{n+1}+\frac{\Delta t}{\rho r_{i}\left(\theta_{j+1}-\theta_{j}\right)} \Delta P_{i, j}^{l},
\end{aligned}
$$

etc. for all subsequent iterations. A surface cell pressure increment is found by linear interpolation or extrapolation of the nearest full cell pressure increment. The procedure for surface cells is discussed in detail in Appendix $D$.

In this iteration technique, we are effectively calculating the net excess mass flow into or out of a $c F .17$ and adjusting the pressure and the four surrounding velocity components accordingly to give zera velocity divergence. In doing so, the divergence of the neighboring cells is affected, and as a result the mesh must be swept iteratively to allow the solution to relax. Iterations are continued unt 11 the maximum cell divergence in the mesh drops below a specified limit. This limit is chosen so that the excess mass flow in any cell due to nonzaro velocity divergence is less than $0.01 \%$ of the total mass in the cell. For this problem a convergence limit of $0.0001 / \Delta t$ is used. When all cells have converged, the velocity solution with correct vorticity and vanishing divergence is achieved. At this point, we have the solution for pressure and velocity at the new time step and we proceed to calculate the temperature field explicitly from equation 12.

Because we are calculating pressure at the cell center based on the cell velocity divergence, we are not required to apply explicit boundary conditions on pressure. This is one advantage of this solution 
technique that is possible because velocity variables are positioned on cell faces and pressure at the cell center.

\section{FINITE-DIFFERENCE BOUNDARY CONDITIONS}

Figure 7 shows a portion of the finite-difference mesh with cell flags and free surface location indicated. Cell flagging will be discussed in the next section. A surrounding layer of boundary cells facilitates boundary conditions by allowing a fictitious tangential velocity outside the watl. The fictitious outside velocity is necessary because of the location of variables as shown in Fig. 3. For the variable mesh, boundary cells are the same size as adjacent cells inside the wall.

The outside velocity is calculated to give zero tangential component at the wall for a no-slip condition. Using mass flow weighted averaging as discussed in Appendix $A$, the outside velocity for a no-sifp wall is found according to

$$
v_{\text {Dut }}=-v_{\text {in }}\left(\frac{r_{\text {in }}}{r_{\text {DUt }}}\right) \text {. }
$$

For the free-slip condition the tangential stress must be zero at the wall. The tangential stress in spherical coordinates is

$$
\tau_{r \theta}=\mu\left(r \frac{\partial}{\partial r}(v / r)+\frac{1}{r} \frac{\partial u}{\partial \theta}\right) .
$$

At a wall with zero normal velocity the tangential stress reduces to 


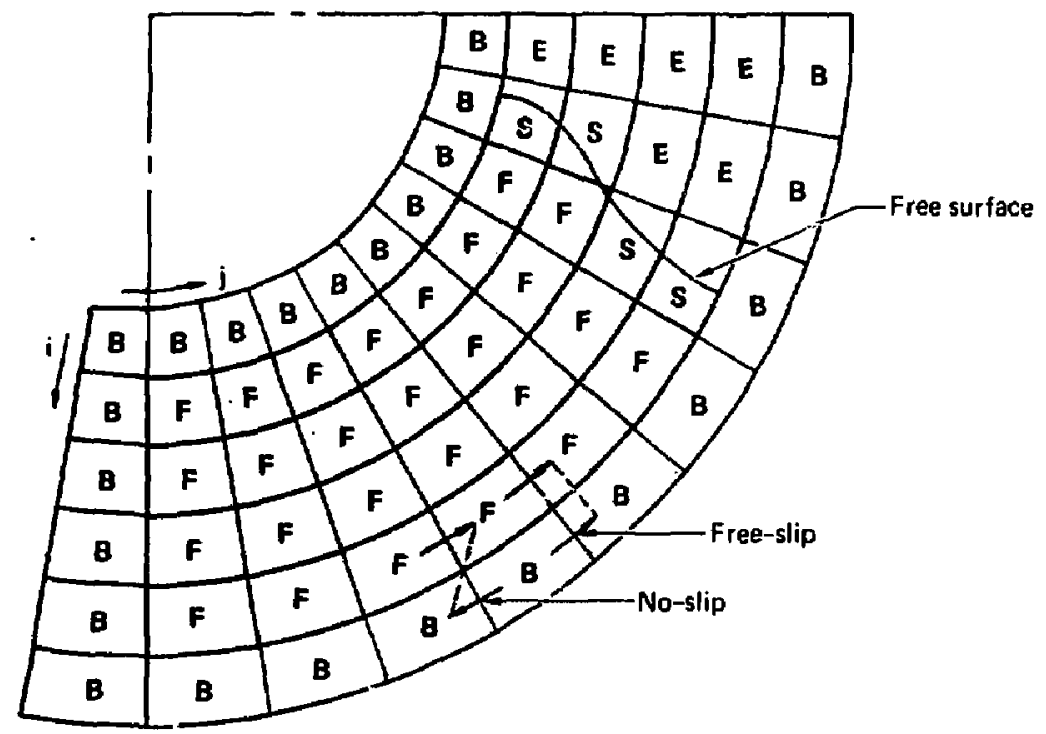

Figure 7. Spherical annulus computing mesh with cell flags. 


$$
\frac{\partial}{\partial r}(v / r)=0
$$

This condition dictates the value of the outside velocity. The outside velocity for a free-slip wall is given by

$$
v_{\text {out }}=v_{\text {in }}\left(\frac{r_{\text {out }}}{r_{\text {in }}}\right) .
$$

\section{FREE SURFACE TREATMENT}

The presence of a free surface makes this a unique fluid mechanics problem. The free surface is an uncontined moving boundary requiring special treatment. The Marker-and-Cell computational method was developed to handle the free surface and all its complexities (14). Cell flagging, marker particles and the treatment of surface cells characterize the MAC method. Massless marker particles mark the fluid and a cell flagging scheme flags cells as full, empty, or surface. Several improvements have been added since the method was first introduced.

Changes and additions to the MAC free surface treatment are required for solution of this problem. There are four specific areas in which the treatment here differs from classical MAC methodology. They are: 1) multiple surface chains for breaking waves, 2) surface adjustment by nonzero divergence in surface cells, 3) special surface or impact cells, and 4) multiple surface cell pressure interpolation from a single full cell. In Appendix D, these features and the application of the standard MAC methodology to our problem are described. 


\section{COMPUTATIONAL RESULTS}

The purpose of this section is to present computational results that demonstrate the capability of the solution technique and represent the major characteristics of the fluid-flow and heat transfer during spherical annulus filling. Computational results for velocity, temperature, and inner sphere heat flux are presented for a spherical annulus with a $0^{\circ} \mathrm{C}$ isothermal inner sphere, an insulated outer sphere, $50^{\circ} \mathrm{C}$. initial annulus water temperature and 20 second $f \nmid 11$ time. Properties of water at $50^{\circ} \mathrm{C}$ were used for these demonstration calculations.

Computations are made with the variable mesh shown in Fig. 8 . There are 16 radial celis, 84 azimuthal cells and 4 cells across the $38.1 \mathrm{~mm}$ diameter inlet. A geometrically stretched mesh is used to improve resolution near the inner sphere and in the inlet region. The radial stretching factor is 1.07 and the azimuthal stretching factor is 1.007. The inner sphere radius of $139.7 \mathrm{~mm}$ and the outer sphere radius of $168.3 \mathrm{~mm}$ were chosen to match a geometry studied by Ward (3), Runde11, et. al. (5), and Bozenan, et. a). (8).

The no-slip velocity condition is applied at the inner sphere where the mesh is fine enough to resolve boundary-layer details. Near the outer sphere, where the mesh is course, the boundary-layer is not resolved and the free-slip condition is applied.

Figure 9 shows calculated azimuthal velocity profiles and velocity vectors at 9 and 19 seconds. These plots illustrate the characteristic flow patterns before and after the free surface passes the point of wall 


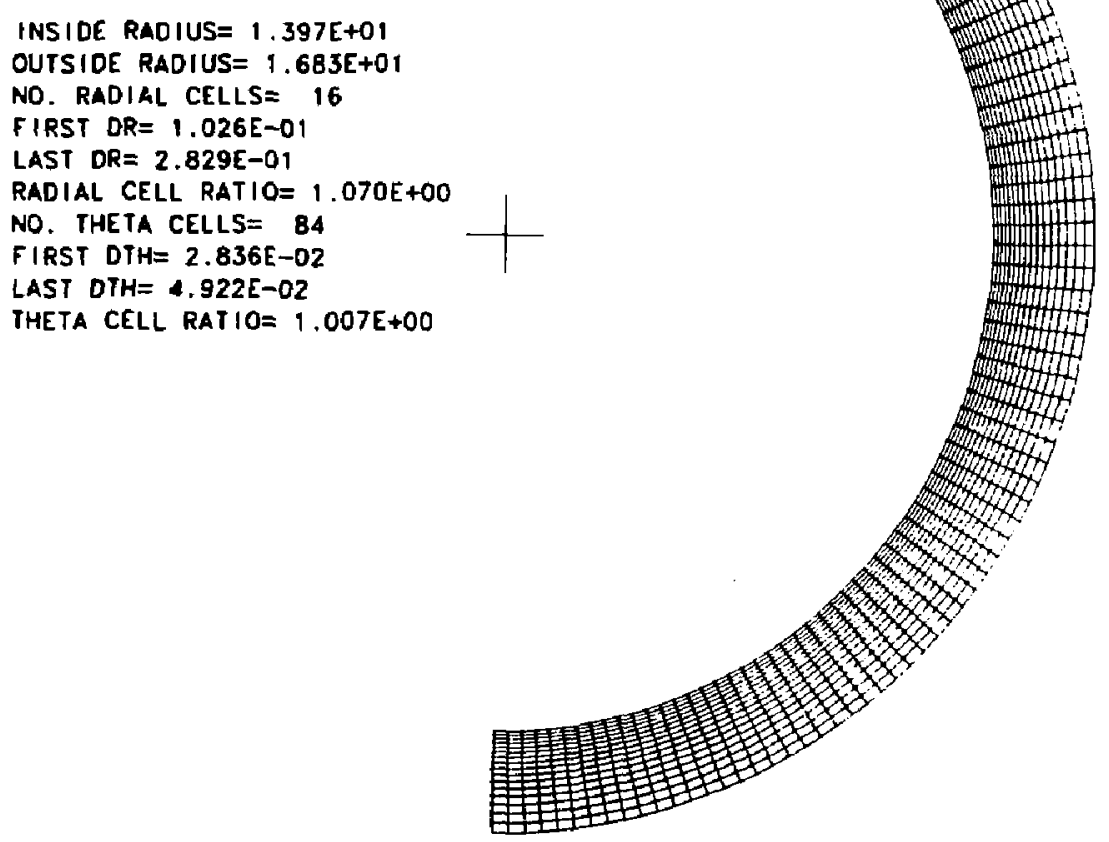

Figure 9. Variable mesh for computing spherical annulus fluid-flow and heat transfer. 


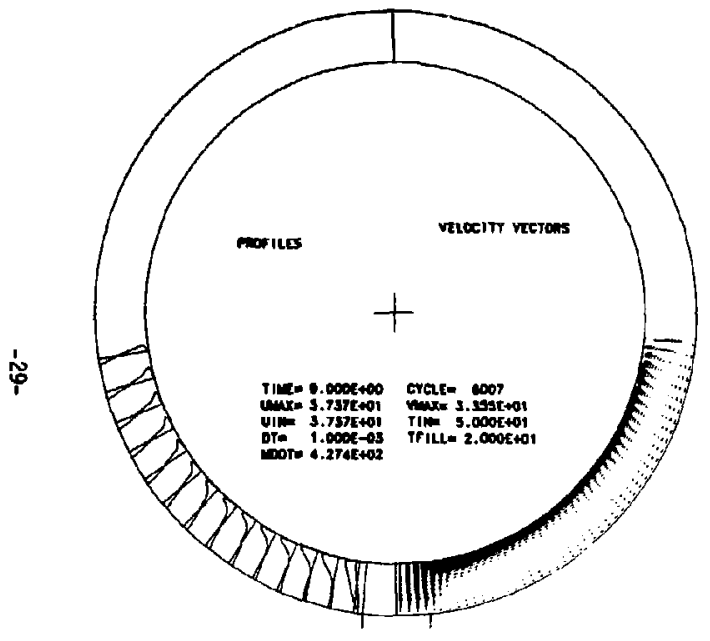

(a) $\mathbf{t}=\mathbf{9}$ seconds

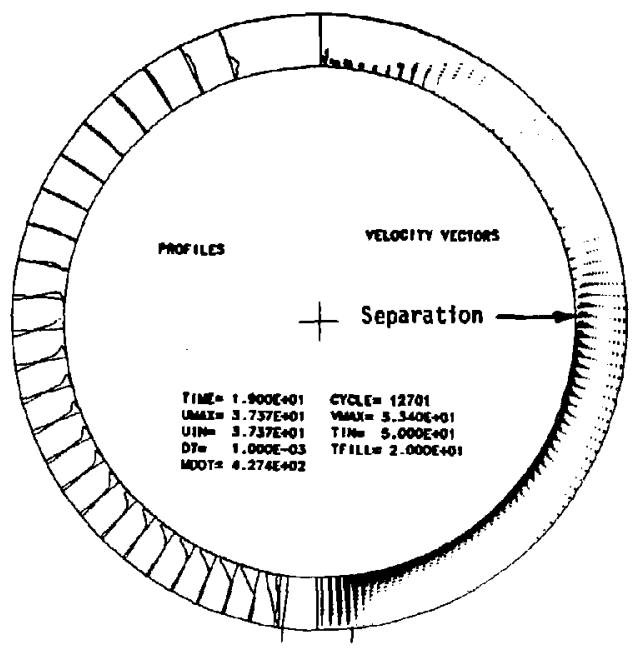

(b) $t=19$ seconds

Figure 9. Aztmuthal velocity profiles and velocity vectors before and after separation. 
jet separation at $\theta=91$ degrees. Velocity vectors and profiles are scaled to the maximum velocity in each case.

In Fig. 9a, the inlet jet impinges on the inner sphere and jets off tangentially, creating a high velocity wall jet near the inner sphere and a uniform low velocity return flow in the outer portion of the annulus. This flow pattern is similar to that observed experimentaliy by Bozeman et al. (8) in the lower portion of the annulus for steady flaw. The flow illustrated in Fig. 9a consists of a main toroidal shaped recir. culation eddy and a very small secondary-eddy at the free surface next to the inner sphere. The secondary-eddy is small and barely visible in the figure. The main recirculation pattern is formed almost immediately when filling begins and simply grows as the annulus fills. The secondary-eddy is created when the surface passes $\theta=50$ degrees. It moves a? ong with the free surface next to the inner sphere until reaching the stationary separation point at $\theta=91$ degrees. Separation is identified by an inflection in the velocity profile followed by flow reversal near the inner sphere.

Figure 9b illustrates the typical flow pattern after the surface has passed the separation point. The toroidal shaped recirculation eddy and the wall jet become steady and no longer grow in length. The steady wall jet thickness decreases initially until about $\theta=30$ degrees, remains approximately constant from 30 to 60 degrees, and then thickens until eventually separting at $\theta=91$ degrees. Downstream of separation, secondary-flow eddies are formed near the inner sphere. These secondary flows and their effect will be seen more clearly in the temperature results. Flow velocities beyond the initial separation point are nearly 
uniform across most of the annulus and are in general small compared to the infet velocity. This result is consistent with experimentai observations from Refs. (3). (5) and (8) for steady flow in a spherical annulus. During most of the filling process, transient behavior is limited to the immediate region of the free surface and the remainder of the flow exhibits quasi-steady behavior.

Computed temperature and pressure contours at 9 and 19 seconds are shown in Fig. 10. The stair-step effect at the surface is caused by the finite cell sizes. Before the surface passes the separation point, temperature is uniform across the annulus except for a thin layer near the inner sphere as shown in Fig. 10a. The relatively high velocity of the wall jet creates a thin thermal boundary-layer that appears as a blackened layer near the inner sphere. The thermal boundary layer is thinnest in the impingement region near $\theta=0$ and thickens to a maximum at separation. The secondary-flow eddy is clearly visable in the temperature contours shown in Fig. 10a. Downstream of separation, velocities and heat transfer rates near the inner sphere vary considerably and are much lower than upstream. Separation and the presence of secondary-flow eddies have a major effect on the temperature contours as shown in Fig. $10 b$.

Computed temperature contours at various times after the surface has passed the separation point are shown in Fig. 11. The sequence illustrates the development of temperature profiles with time. At $t=$ 19.95 seconds, the flow next to the inner sphere reverses direction at three locations creating three eddies. The first flow reversal occurs at $\theta=91$ degrees where the wall jet separates. There is a reattachment 


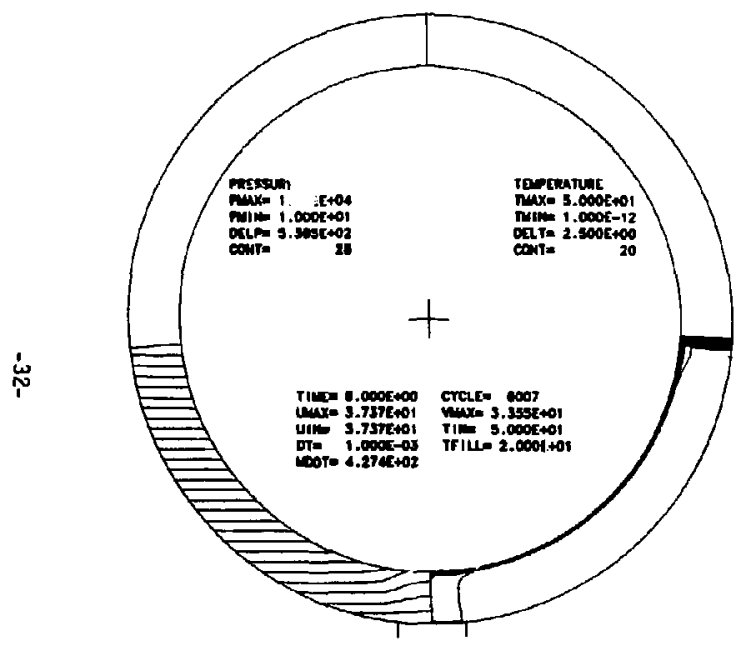

(a) $t=9$ seconds

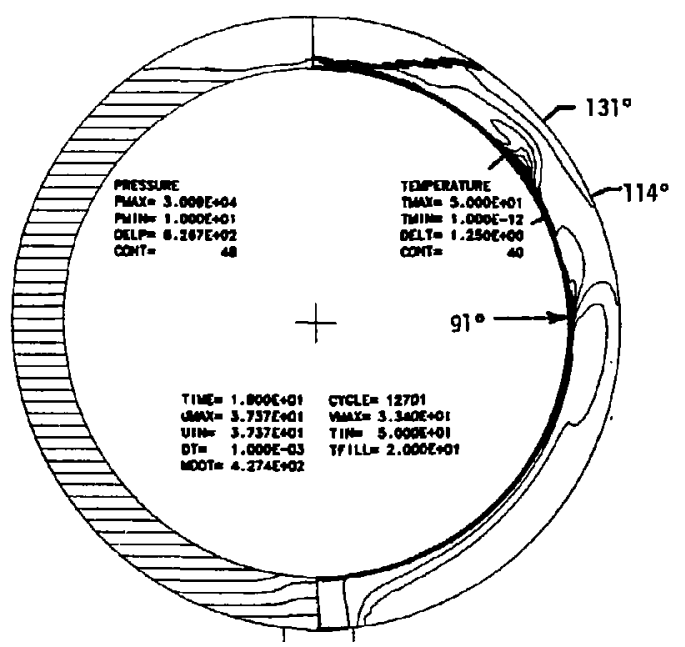

(b) $t=19$ seconds

Figure 10. Pressure and temperature contours before and after separation. 


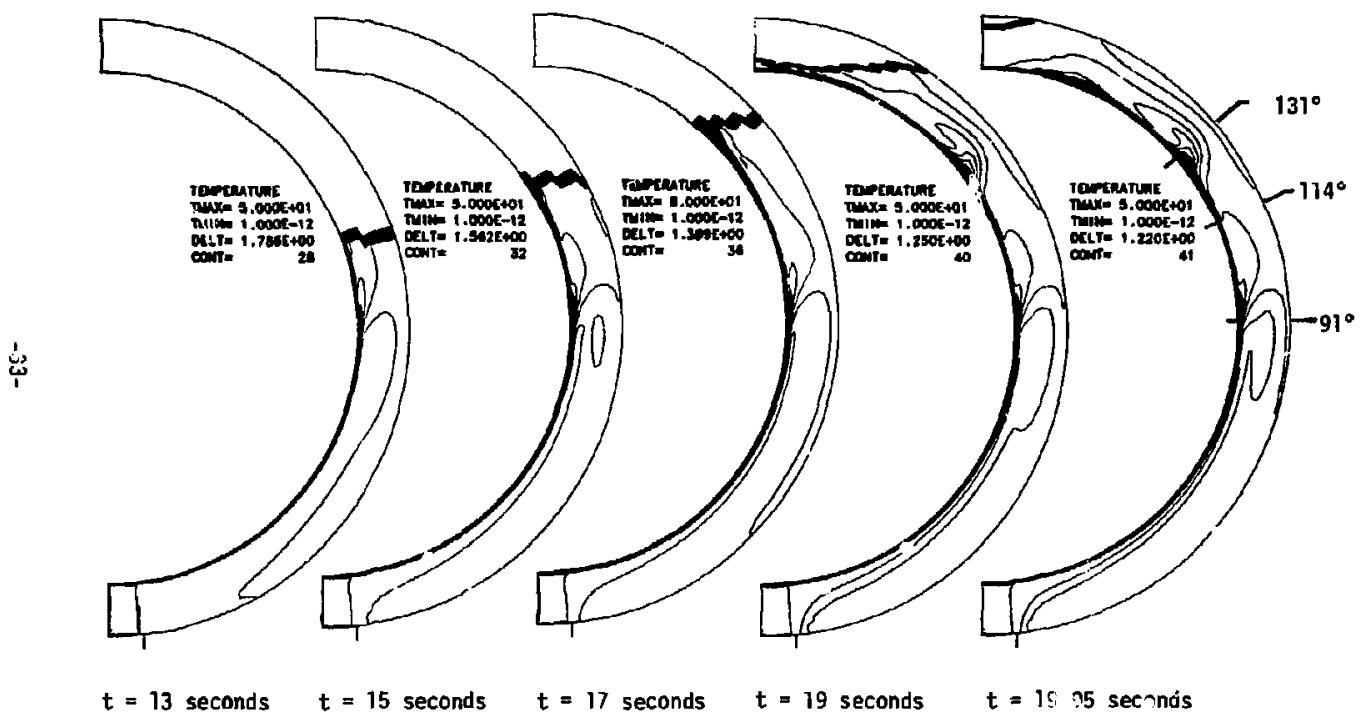

Figure 11. Temperature contours at various times after the surface has passed the separation point at $\theta=91$ degrees. 
point at $\theta=114$ degrees and another separation point at $0=13$ !

degrees. Locations of these points are indicated in Fig. 11. The isotherms point out the effect of the secondary-flow eddies on the temperature field. The eddies in the upper half of the annulus were probably not visible to Ward (3) or Rundell et. al. (5) because dye diffusion obscured vision of any flow details in that region.

Figure 12 is a plot of inner sphere heat flux rate versus angle at times of $5,10,15$, and 19.95 seconds. Separation and the presence of secondary-flow eddies create the peaks and valleys in the heat flux rate curves. The sudden increase in heat flux rate at the ends of the 5 and 10 second curves is caused by the presence of the small eddy near the surface. This eddy and the resultant effect on the heat flux rate move with the surface until reaching the separation point at $\theta=91$ degrees. After the surface passes this point the edidy begins to grow in length until the flow reattaches at $\theta=114$ degrees. The growing eddy causes a decrease in heat flux rate from $t=15$ to 19.95 seconds between $\theta=91$ and 114 degrees. Three secondary-flow eddies are eventually formed and each one criates a separate valley in the heat flux rate curve as seen in Fig. 12 at $t=19.95$ seconds. The final increase in heat flux rate beyond $\theta=160$ degrees at $t=19.95$ seconds is the resu, $t$ of increased flow velocities near the inner sphere. This incrcase is a reversal in the initial generat downward trend in heat flux rate.

During the majority of the filling process, transient velocities are limited to the immediate region of the free surface and the remainder of the flow exhibits quasi-steady behavior. This is especially true after the surface passes the separation point. As a resist, we expect to 


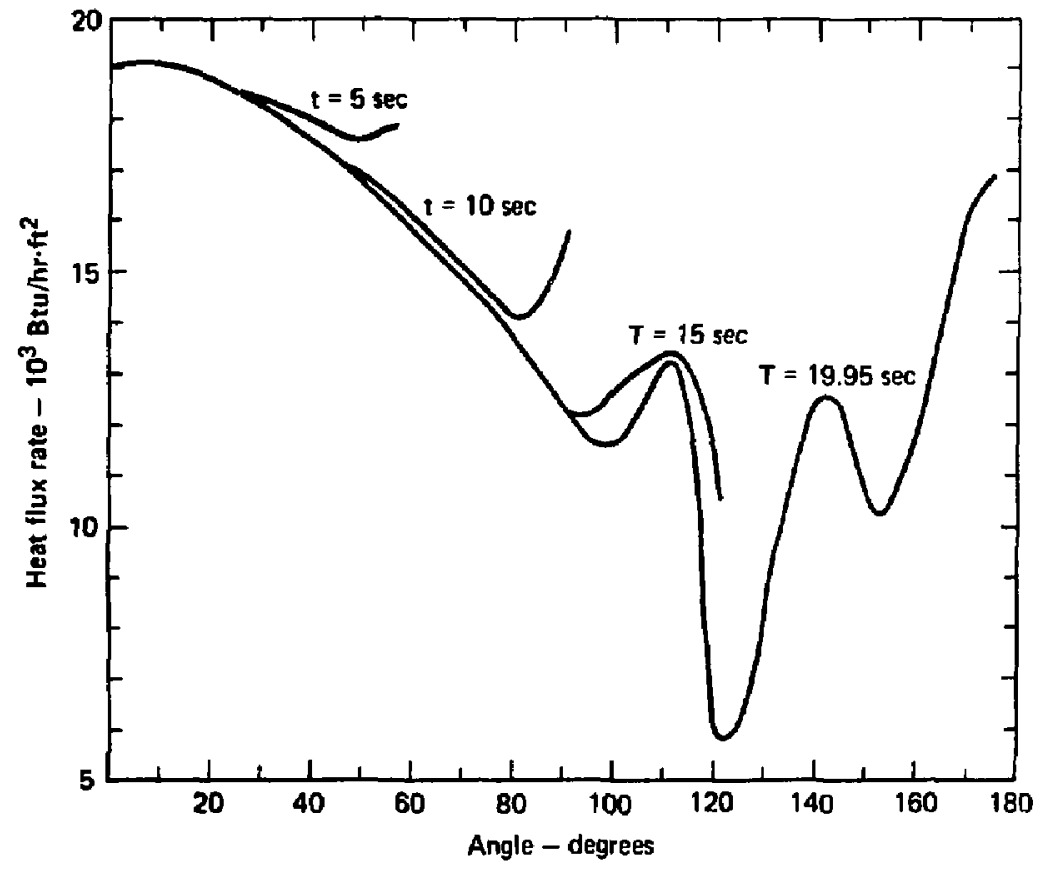

Figure 12. Inne' sphere heat flux rate versus angle at various times. 
be able to compare our computed separation point at $D=91$ degrees to the experimental observations of others for steady flow.

Runde 71 (4) and Runde !l et. aT. (5) reported a steady separation point between $0=45$ and 50 degrees. The difference in the computed separation point and the experiments can be attributed to the lack of turbulence modeling in the calculations. The main effect of a turbuience model on the calculations would be an increase in the effective fluid viscosity. Effective turbulent viscosity varies with flow properties and can easily be one hundred times larger than the laminar viscosity.

To gel an idea of the effect of increased effective viscosity on the flow pattern, a test case was run with laminar viscosity ten times the viscosity used in the previous calculations. Azimuthal velocity profiles and temperature contours for the high viscosity calculation are shown in Fig. 13. The wall jet has separated at $\theta=56$ degrees, indicating that the separation point location is affected by the fluid viscosity in these problems. We expect to see the same qualitative shift in calculated separation point location due to the increase in effective viscosity from a turbulence model. 


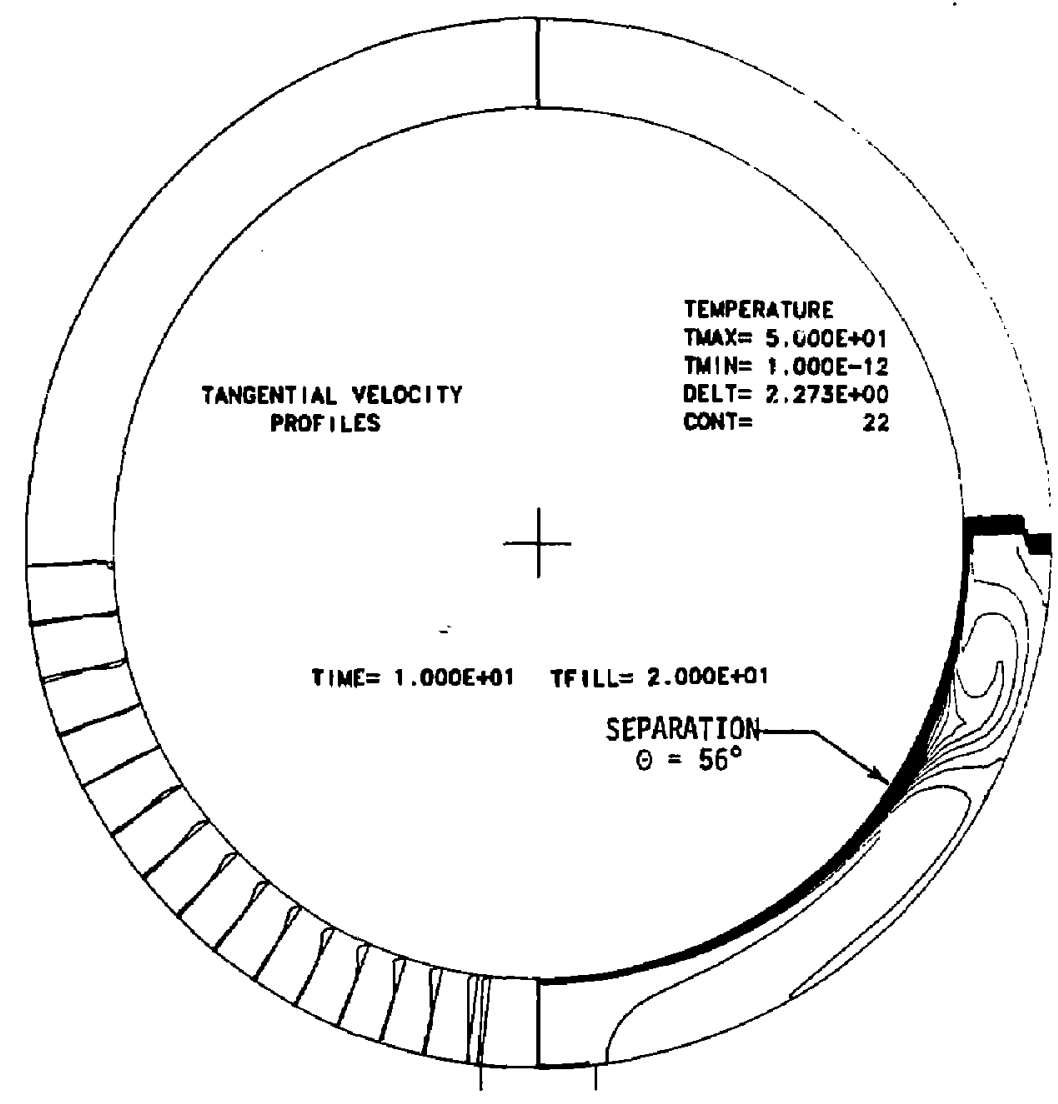

Figure 13. Velocity profiles and timperature contours at $t=10$ seconds for laminar viscosity 10 times larger than water 


\section{SUMMARY AND CONCLUSIONS}

In this paper, a computational technique for calculating laminar fluid velocities and temperatures during the filling of a spherical annulus has been described. The nature of the free surface flow has required special treatment beyond the standard techniques available for free surface flow problems. Results of velocity, temperature, and heat flux have been presented for a spherical annulus filling problem. These sample results are intended to demonstrate the capability of the computational technique as well as display some of the major and important features of the flow.

Results indicate the impingement region and the wall jet along the inner sphere are the important areas for heat transfer. The location of separation has a major effect on the predicition of tota] wall heat flux and annulus fluid temperatures. Upstream of separation, heat is extracted from the annulus fluid as it flows along the inner sphere. The total amount of heat extracted is directly dependent upon the length of the flow path along the inner sphere which is determined by the location of the separation point. Fter separation, the main flow moves to the outer sphere and velocities and heat transfer rates near the inner sphere are lower than in the wall jet region. Large changes in heat flux rate as a function of angle are present downstream of separation and have been shown to be a result of secondary-flow eddies near the inner sphere.

An important feature of the flow, is the quasi-steady behavior in all regions except the immediate vicinity of the free surface. This fact allows us to compare qualitative calculated features of the filling problen to the results of steady flow experiments from the literature. 
The computed : low pattern upstream of separation agrees qualitatively with experimental results in the literature for steady spherical annulus flow. The fact that the main flow moves to the outer sphere downstream of separation with nearly stagnant flow near the inner sphere has also been observed experimentally for steady flow and reported in the literature $(3,5,8)$. Downstream eddies near the inner sphere were probably not seen in experiments because of reduced visual resolution due to dye diffusion.

The major difference between transient calculations and the steady flow experimental results is the location of wall jet separation. The calculations indicate separation at $0=91$ degrees compared to experimental observation of separation between $\theta=45$ and 50 degrees $(4,5)$. Turbulence in the flow has a major effect on the lacation of separation. We have shown that increasing the laminar viscosity causes the calculated separation point to move downstream in closer agreement with experi. ments. The increased effective viscosity of a mixing length (or ather) turbulence model will have the same qualitative effect on separation as the increased laminar viscosity.

Calculations for slow filling in the laminar flow regime and the addition of a turbulence model aimed primarily at modeling the wall jet region are planned. The wall jet and impingement region are the important areas for heat transfer and this is where we plan to focus the attention of the turbulence modeling. The quasi-steady boundary-layer like nature of the wall jet makes mixing length turbulence models look promising for this region. He plan to consider mixing length and other models for the impingement and downstream regions. 


\section{REFERENCES}

1. M. H. Cobble, Spherical Shell Heat Exchanger - Dirichlet Problem,

J. Franklin Institute, Sept. 1963, p. 197.

2. R. B. Bird, W. E. Stewart and E. N. Lightfoot, Transport

Pehnomena, (Wi ley, New York, 1964), p. 117.

3. E. G. Ward, Flow Through the Annu lus Formed Between Concentric

Spheres, Masters Thesis, University of Houston, Houston, Texas (1966).

4. H. A. Runde 11, Forced Convection in Concentric Sphere Heat

Exchangers, Ph.D. Thesis, University of Houstan, Houston, Texas (1966).

5. H. A. Runde11, E. G. Ward and J. E. Cox, "Forced Convection in Concentric-Sphere Heat Exchangers," J. Heat Trans., Transactions ASME, Ser. C, Vol. 90, (1968), p. 125.

6. J. E. Cox and B. K. Sahni, "The Concentric-Sphere Heat Exchanger," J. Heat Trans., Transactions ASME, Ser. C, Vol. 93, (1971), p. 468.

7. J. D. Bozeman, A Flow Visualization Study of the Flow in the Entrance Region of an Annulus Formed between Concentric Spheres, Masters Thesis, University of Houston, Houston, Texas (1968).

B. J. D. Bozeman and C. Dalton, "Flow in the Entrance Region of a Concentric Sphere Heat Exchanger," J. Heat Trans., Transactions ASME, Ser. C, Vol. 92, (1970), p. 184.

9. J. R. Brown, Natural Convection Heat Transfer between Concentric Spheres, Ph.D. Thesis, University of Texas, Austin, Texas (1967). 
10. K. N. Astill, "An Analysis of Laminar Forced Convection between Concentric Spheres," J. Heat Trans., Transactions ASME, Ser. C, Vol. $98,(1976)$, D. 601 .

11. F. H. Harlow and J. E. Welch, "Numerical Calculation of Time-Dependent Viscous Incompressible Flow of Fluid with Free Surface," Phys. Fluids, Vol. 8, (1965), p. 2182.

12. W. F. Hughes and E. W. Faylord, Basic Equations of Engineering Science (Schaum Publishing, New York, 1964).

13. P. J. Roache, Computational Fluid Dynamics (Hermosa Publishers, Albuquerque, New Mexico, 1972).

14. J. E. Weich, F. H. Harlow, J. P. Shannon and B. J. Daly, The MAC Method: A Computing Technique for Solving Viscous, Incomeressible, Transient Fluid-Flow Problems Involving Free Surfaces, Los Alamos Scientific Laboratory Report LA-3425 (1966).

15. C. W. Hirt, B. D. Nichols and N. C. Romero, SOLA -- A Numerical Solution Algorithm for Transient Fluid Flows, Los Alamos Scientific Laboratory Report LA-5852 (?975).

16. A. J. Chorin, "Numerical Solution of the Navier-Stokes Equations," Math. Comp., Vol. 22, (1968), p. 745.

17. J. A. Viecel1;, "A Method for Including Arbitrary External Boundaries in the MAC Incompressible Fluid Computing Technique," J. Comp. Phys., Vol. 4, (1969), p. 543.

18. C. W. Hirt and J. L. Cook, "Calculating Three-Dimensional Flow Around Structures and Over Rough Terrain," J. Comp. Phys., Vol., 10, $(1972)$, p. 324 .

19. H. F. Ames, Numerical Methods for Partial Differential Equations (Barnes and Noble Publishing, New York, 1969). 


\section{APPENDIX A}

\section{NONSTANDARD TERMS FOR FINITE-DIFFERENCE EQUATIONS}

The finite-difference momentum and energy equations in Section IV (equations 10, 11, and 12) contain terms that do not lie on the defined mesh point locations as shown in Fig. 3. In past MAC applications, these terms have been calculated by linear averaging for cartesian or cylindrical geometry. Linear averaging in the radial direction of cylindrical coordinates introduces a small error which has been neglected in past app lications.

In spherical coordinates, cell face areas change in every coordinate direction and linear averages can be improved by accounting for area changes. We account for area changes by calculating velocities for nonstandard locations from a linear average of mass flow. For incompressible flow this is equivalent to averaging the product of velocity and area.

Below is a list of nonstandard velocity terms from equat ion 10 (the radial momentum equation) with subscripts referenced to cell $(i, j)$ as in Fig. 3, Section IV $v T=v_{i+1 / 2, j+1 / 2}^{n}=\left\{\frac{v_{i, j+1 / 2}^{n} r_{i} \Delta r_{i}+v_{i+1, j}^{n}+1 / 2 r_{i+1} \Delta r_{i+1}}{2 r_{i+1 / 2} \Delta r_{i+1 / 2}}\right\}$, $V B=v_{i+1 / 2, j-1 / 2}^{n}=\left\{\frac{v_{i, j-1 / 2}^{n} r_{i} \Delta r_{j}+v_{i+1, j-1 / 2}^{n} r_{i+1} \Delta r_{i+1}}{2 r_{i}+1 / 2 \Delta r_{j+1 / 2}}\right\}$, $u T=u_{i+1 / 2, j+1 / 2}^{n}=\left\{\frac{u_{i+1 / 2, j}^{n} \sin \theta_{j} \Delta \theta_{j}+u_{i+1 / 2, j+1}^{n} \sin \theta_{j+1} \Delta \theta_{j+1}}{2 \sin \theta_{j+1 / 2} \Delta \theta_{j+1 / 2}}\right\}$, 


$$
\begin{gathered}
U B=u_{i+1 / 2, j-1 / 2}^{n}=\left\{\frac{u_{i+1 / 2, j}^{n} \sin \theta_{j} \Delta \theta_{j}+u_{i+1 / 2, j-1}^{n} \sin \theta_{j-1} \Delta \theta_{j-1}}{2 \sin \theta_{j-1 / 2} \Delta \theta_{j-1 / 2}}\right\}, \\
U R=u_{i+1, j}^{n}=\left\{\frac{\left(u_{i+1 / 2, j}^{n} r_{i+1 / 2}^{2}+u_{i+3 / 2, j}^{n} r_{i+3 / 2}^{2}\right.}{2 r_{i+1}^{2}}\right\}, \\
U L=u_{i, j}^{n}=\left\{\frac{u_{i+1 / 2, j}^{n} r_{i+1 / 2}^{2}+u_{i-1 / 2, j}^{n} r_{i-1 / 2}^{2}}{2 r_{i}^{2}}\right\}, \\
\quad v_{i+1 / 2, j}^{n}=\left\{\frac{\sin \theta_{1+1 / 2} v T+\sin \theta_{j-1 / 2} v B}{2 \sin \theta_{j}}\right\} .
\end{gathered}
$$

Nonstandard terms for equation 11 (the azimuthal monentum equation) are

$$
\begin{aligned}
& U R=u_{i+1 / 2, j+1 / 2}^{n}=\left\{\frac{u_{i+2 / 2, j}^{n} \sin \theta_{j} \Delta \theta_{j}+u_{i+1 / 2, j+1}^{n} \sin \theta_{j+1} \Delta \theta_{j+1}}{2 \sin \theta_{j+1 / 2} \Delta \theta_{j+1 / 2}}\right\}, \\
& U L=u_{i-1 / 2, j+1 / 2}^{n}=\left\{\frac{u_{i-1 / 2, j}^{n} \sin \theta_{j} \Delta \theta_{j}+u_{i-1 / 2, j+1}^{n} \sin \theta_{j+1} \Delta \theta_{j+1}}{2 \sin \theta_{j+1 / 2} \Delta \theta_{j+1 / 2}}\right\} \\
& V T=v_{i, j+1}^{n}=\left\{\frac{v_{i, j+3 / 2^{n i n} \theta_{j+3 / 2}+v_{i, j+1 / 2}^{n} \sin \theta_{j+1 / 2}}^{2 \sin \theta_{j+1}}}{2},\right. \\
& v B=v_{1, j}^{n}=\left\{\frac{v_{i, j+3 / 2}^{n} \sin \theta_{j+3 / 2}+v_{i, j+1 / 2}^{n} \sin \theta_{j+1 / 2}}{2 \sin \theta_{j+1}}\right\},
\end{aligned}
$$

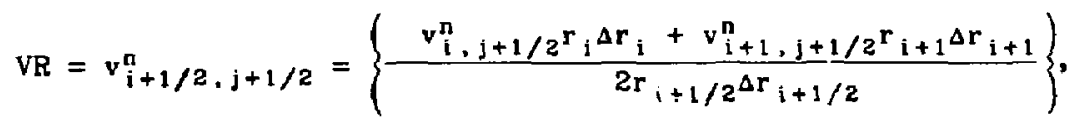




$$
\begin{gathered}
v L=v_{i-1 / 2, j+1 / 2}^{n}=\left\{\frac{v_{i, j+1 / 2}^{n} r_{i} \Delta r_{i}+v_{i-1, j+1 / 2}^{n} r_{i-1} \Delta r_{i-1}}{2 r_{i}-1 / 2 \Delta r_{i-1 / 2}}\right\}, \\
u_{i, j+1 / 2}^{n}=\left\{\frac{r_{i+1 / 2}^{2} U R+r_{i-1 / 2}^{2} U L}{2 r_{j}^{2}}\right\} .
\end{gathered}
$$

The nonstandard terms used in equations 12 (the energy equation) are calculated by linear averaging

$$
\begin{aligned}
& T L=T_{i-1 / 2, j}^{n}=\frac{1}{2}\left(T_{i, j}^{n}+T_{i-1, j}^{n}\right), \\
& T B=T_{i, j-1 / 2}^{n}=\frac{1}{2}\left(T_{i, j}^{n}+T_{i, j-1}^{n}\right), \\
& T R=T_{i+1 / 2, j}^{n}=\frac{1}{2}\left(T_{i, j}^{n}+T_{i+1, j}^{n}\right), \\
& T T=T_{i, j+1 / 2}^{n}=\frac{1}{2}\left(T_{i, j}^{n}+T_{i, j+1}^{n}\right)
\end{aligned}
$$




\section{APPENDIX B \\ NUMERICAL STABILITY CONDITIONS}

The finite-difference momentum and energy equations (equations 10, 11, and 12, Section IV) are restricted by numerical instabilities to a maximum allowable time step. Attempts by many authors to analyze the numerical stability conditions of general nontinear finite-difference equations have failed to provide both sufficient and necessary conditions that insure numerical stability. Some of the methods reported in the literature provide necessary stability conditions that can be used as a guideline (Bi). The final test, however, lies in numerical experimentation using available stability conditions as guidelines.

The most straight-forward and generally accepted method of stability analysis is the von Neumann method for linearized equations. A discussion of the von Neumann method is presented by Roache (B2). In the von Neumann analysis, the equations are linearized by assuming constant coefficients in the convection terms and the solution is expanded in terms of Faurier components. For our momentum and energy equations the Fourier components are

Radial Momentum:

$$
u_{i+1 / 2, j}^{n}=\psi^{n} \exp 1\left(i+1 / 2 \phi_{r}+j \phi_{\theta}\right)
$$

Azimuthal Momentum:

$$
v_{i, j+1 / 2}^{n}=\psi^{n} \exp I\left(i \phi_{r}+j+1 / 2 \phi_{\theta}\right)
$$




\section{Energy Equation:}

$$
T_{i, j}^{n}=\psi^{n} \exp I\left(i \phi_{T}+j \phi_{0}\right)
$$

In the above equations, $\psi^{n}$ is the amplitude function at time level $n$ for the particular component with phase angle $\Phi$ and $I=(-1)^{1 / 2}$. Positive convection velocities are assumed for upwind differencing terms with $z=$ 1 and the above Fourier components are substituted into the finite-difference equations. The amplification factor

$$
G=\frac{\psi^{n+1}}{\psi^{n}}
$$

is introduced to define stability and the stability criteria

$$
|\mathbf{c}|<1 \text {, }
$$

must be satisfied for all possible $\phi_{r}$ and $\phi_{\theta}$. The resulting stability conditions for the linearized momentum and energy equations are Radial Momentum Stability Condition:

$$
\Delta t<\left\{\frac{1}{\left|\frac{u}{\Delta r}\right|_{\max }+\left|\frac{v}{r \Delta \theta}\right|_{\max }+\left|\frac{2 \nu}{r^{2} \Delta \theta^{2}}\right|_{\max }}\right\} \text {, }
$$

\section{Az imuthal Momentum Stability Condition:}

$\Delta t<\left\{\frac{1}{\left|\frac{u}{\Delta r}\right|_{\max }+\left|\frac{v}{r \Delta \theta}\right|_{\max }+\left|\frac{u}{2 r}\right|_{\max }+\left|\frac{2 \nu}{\Delta r^{2}}\right|_{\max }}\right\}$ 


\section{Energy Equation Stability Condition:}

$$
\Delta t<\left\{\frac{1}{\left|\frac{u}{\Delta r}\right|_{\max }+\left|\frac{v}{r \Delta \theta}\right|_{\max }+2 a\left[\frac{1}{\Delta r^{2}}+\frac{1}{r^{2} \Delta \theta^{2}}\right]_{\max }}\right\}
$$

The above expressions limit the convective plus difrusive fluxes to less than a cell width in a sing e cycle. In our problem, the convective flux limitation, of ten called the Courant condition, is the dominant factor.

The results of the von Neumann stability analysis provide necessary stability conditions for the nonlinear equations but do not in general predict nonlinear instabilities. Experience in computing with equations 10, 11, 12, and 13 has shown the equations are generaliy stable within the conditions of the linear von Neumann analysis. The numerical viscosity effect of donor cell differencing of convective terms plays a large role in damping out instabilities and stabilizing the numerical solution. 


\section{REFFERENCES FOR APPENDIX B}

B]. C. H. Hirt, "Heuristic Stability Theory for Finite-Difference Equations," J. Comp. Phys., Vor. 2, (1968), p. 339.

B2. P. J. Roache, Computational Fluid Dynamics (Hermosa Publishers, Albuquerque, New Mexico, 1972). 


\begin{abstract}
APPENDIX $\mathrm{C}$
CONSERVATION OF VORTICITY BY PRESSURE-VELOCITY ITERATIONS, THE POISSON EQUATION FOR PRESSURE, AND THE MAC CORRECTIVE TERM

The purpose of this appendix is: 1) to demonstrate that the numerical iterations on pressure and velocity presented in Section IV conserve vorticity, 2) to show that the iterative procedure is equivalent to solving the Poisson equation for pressure and 3) to prove the existence of the MAC corrective term in the numerical solution technique.
\end{abstract}

\title{
ITERATIVE VORTICITY CONSERVATION
}

In Section IV, we discussed how the numerical iterations achieve mass conservation by adjusting pressures according to the cell velocity divergence and then adjusting velocities to reflect the new pressure gradient. The velocity field was iteratively adjusted by effectively adding tiie gradient of the scalar pressure field to each velocity component in the fashion

$$
\overrightarrow{\mathrm{u}}^{\mathrm{I}+1}=\overrightarrow{\mathrm{u}}^{\mathrm{I}}+\mathrm{C}_{0} \overrightarrow{\nabla P}^{I}
$$

where:

$$
C_{0}=\frac{\Delta t}{\rho}
$$

From this equation and the definition of vorticity $(2 \Lambda=\vec{\nabla} \times \overrightarrow{0})$, we can calculate the vorticity of the new velocity field as 


$$
2 A^{I+1}=\vec{\nabla} \times \vec{U}^{1+1}=\vec{\nabla} \times \vec{U}^{I}+\vec{\nabla} \times\left(\vec{\nabla} P^{1}\right) .
$$

However, the last term is the curl of the gradient which is identically zera (CI). Thus, the new vorticity is equal to the original vorticity

$$
2 \Lambda^{1+1}=\vec{\nabla} \times \vec{U}^{1+1}=\vec{\nabla} \times \vec{U} !,
$$

and the solution has preserved vorticity.

POISSON PRESSURE EQUATION

The iterative technique is exactiy equivalent to solving the Poisson equation for pressure, as was done in the original MAC treatment. We will illustrate this by going back to equation 4 in Section IV, and lumping terms together to get the equation

$$
\frac{\partial \vec{U}}{\partial t}=\vec{\Gamma}_{i}^{n} . j-\frac{\vec{\nabla} P}{\rho},
$$

where:

$$
\vec{r}_{i, j}^{n}=-\vec{\nabla} \cdot(\vec{u} \vec{u})-\vec{\nabla} \times[\nu(\vec{\nabla} \times \vec{U})]+\vec{g} .
$$

Taking the divergence of equation C-4 and pulting the time derivative outside of the divergence, since the spatial coordinates are independent of time, we have 


$$
\frac{\partial}{\partial t}(\vec{\nabla} \cdot \vec{j})=\vec{\nabla} \cdot \vec{r}_{1}^{n}, j-\frac{\vec{\nabla}^{2} P}{p} .
$$

To prevent error accumulation due to nonzero velocity divergence the additional constraint

$$
\frac{\partial}{\partial l}(\vec{\nabla} \cdot \vec{U})=0,
$$

is imposed. With this constraint equation C-6 reduces to the Poisson equation for pressure

$$
\frac{\vec{\nabla}^{2} P}{\rho}=\vec{\nabla} \cdot \vec{\Gamma}_{i, j}^{n} \cdot
$$

Another way of showing this is by substituting equations 14 into equation 13 in Section IV and rearranging to find the resulting expression is exactly the finite-difference equivalent of the above Poisson equation.

MAC CORRECTIVE TERM

Our solution technique contains the MAC corrective term to prevent errors due to the finite convergence limit from accumulating from time step to time step (C2). To illustrate the corrective term we go back to equation $\mathrm{C}-4$ and write the finite-difference form for the time derivative

$$
\frac{\vec{U}_{i, j}^{n+1}-\vec{U}_{i, j}^{n}}{\Delta t}=\vec{r}_{i, j}^{n}-\frac{\overrightarrow{\nabla P}}{\rho} .
$$


We then solve for $\vec{v}_{i, j}^{n+1}$ and take the divergence to get

$$
\vec{\nabla} \cdot \vec{U}_{i, j}^{n+1}=\vec{\nabla} \cdot \vec{U}_{i, j}^{n}+\Delta t\left(\vec{\nabla} \cdot \vec{\Gamma}_{j, j}^{n}-\frac{\vec{\nabla}^{2} P}{\rho}\right) \cdot
$$

Zero velocity divergence at cycle $n+1$ in equation $C-10$ requires that

$$
\frac{\vec{\nabla}^{2} \underline{p}}{\rho}=\vec{\nabla} \cdot \vec{\Gamma}_{i, j}^{n}+\frac{\vec{\nabla} \cdot \vec{U}_{i, j}^{n}}{\Delta t} .
$$

This equation is the same as equation C-8 with the exception of the correction term $\frac{\vec{v} \cdot \vec{u}_{\mathfrak{i}, j}^{n}}{\Delta t}$. If the solution at cycle $n$ were perfect this term would be exactly zero. Since the iterations proceed only to a given limit, this term will have a small but finite value and will act to correct slightly the $n+1$ time level pressures to account for past errors in divergence. In this way, the divergence error from each cycle is corrected and not allowed to increase with time. 


\section{REFFERENCES FOR APPENDIX C}

C1. H. F. Hughes and E. W. Gaylord, Basic Equations of Engineering Science (Schaum Publishing, New York, 1964).

C2. C. W. Hirt and F. H. Harlow, "A General Corrective Procedure for Mumerical Solution of Initial-Value Problems," J. Comp. Phys., Vol. 2, (1967), p. 114. 


\section{APPENDIX D \\ DETAILS OF FREE SURFACE TREATMENT}

\section{BACKGROUND}

The original MAC method was simplified by Amsden and Harlow in the SMAC method (D1), by adding a more efficient solution of the governing equations and a simplified treatment of pressure at houndaries. Hirt and Shannon (D2), added refinements in the free surface stress conditions for low Reynolds number flow. Chan and Street (03), defined the surface by a single valued height function and eliminated the need for marker particles. In addition, they developed a scheme to use marker partinles only on the surface. Both methods were limited to $10 \mathrm{w}$ amplitude wave motion with a single valued free surface. The surface particle chain reduced the required number of particles from several thousand to a few hundred. This refinement also made precise location of the surface possible allowing accurate conditions on pressure to be applied. Nichols and Hirt (D4), extended the surface marker particle scheme to multiple valued surfaces that result from highly contorted flaws. In addition, Nichols and Hirt improved the free surface stress conditions and discussed their use for higher Reynolds number flow. Hirt et. al. (D5), developed the SOLA code which is capable of calculating incompressible flow in closed spaces and is simplified for use by persons with little or no experience in numerical fluid mechanics. A modified version (SOLA-SURF), handles free surface flow by defining the surface with a single valued surface height function in the manner of Chan et. al. (03). The solution method in SOLA is essentially the same as the method used here. 
For our problem, we use the surface marker scheme and free surface stress conditions of Nichols and Hirt (D4) with modifications to fit our situation. The method and modifications are discussed in more detail in the following sections.

\section{CELL FLAGGING}

Cell flags tell us whether or not a cell contains fluid and indicate which cells contain the free surface. An example of cell flags was shown by Fig. 7, in Section IV. Cells are flagged based on the location of the surface marker particle chain. At the beginning of the filling process, all cells are flagged empty and as fluid enters the annulus, cell flags are adjusted according to the location of the surface. During each cycle after the velocity solution has been obtained, marker particles are moved by linear interpolation of the velocity field and the mesh is swept to update cell flags. On the first sweep, cells containing marker particles are flagged surface. On the second sweep, we check for surface cells that do not contain marker partictes. These celis are flagged full if there is no empty neighbor and empty if an empty neighbor exists. Finally, any cell containing particles and having no empty neighbors is flagged full.

Normally a cell flag would not jump directly from empty to full in

one cycle, however, situations occur in this flagging scheme that cause a cell flag to jump from empty to full. This situation occurs when the inlet jet impacts the inner sphere and also when a wave hits a wall. When a cell jumps from empty to full, there is an apparent net gain in fluid mass which results in the annulus filling too rapidly. Total 
accumulation of extra mass is dependent upon the size of the cells and the number of times this situaiton occurs during the filling process. Figure DI illustrates the situation when the fluid jet impacts the inner sphere.

We define special surface cells to avoid this error near wills. Special surface cells allow the surface to move within less than a cell width of the wall. We have determined the following requirements as necessary for a special surface cell. The cell must be directly adjacent to a solid boundary, contain part of the surface marker chain, and have been flagged fult by the standard flagging routine indicating that it has no empty neighbors. Then, if more than half of the particles in the cell are in the outer one-fifth, the flag is left full indicating the surface has contacted the wall. If the fraction of particles in this layer is less than half, the cell is still eligible to be a special surface cell and the theta coordinate of the chain attachment point is compared to the coordinate of the cell center. If the attachment point has a smaller theta, the cell is flagged as a special surface cell, if not, the flag is left full.

A special surface cell is distinguished by a nonzero normal wall velocity calculated by equation 13, in Section IV, to give zero velocity divergence for the cell. This is the same treatment given a regular surface cell with a single empty neighbor. The effects of the special surface cells are: 1) presence of the wall is not felt until the surface effectively touches it and, 2) artificial mass creation is reduced. 


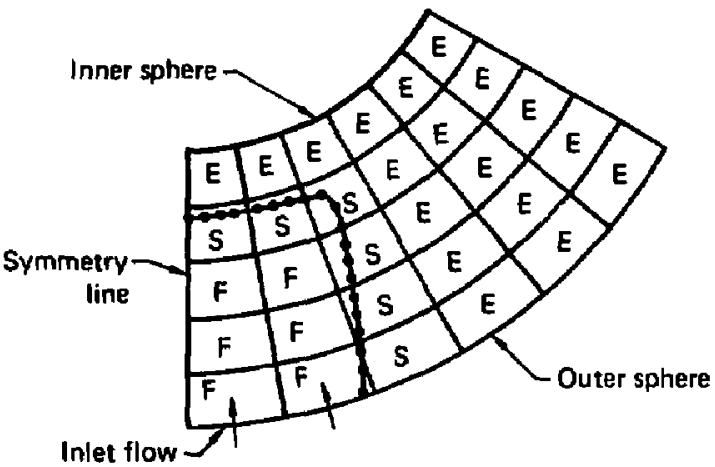

(a) FTuid jet about to enter final Tayer of cells before inner sphere wall.

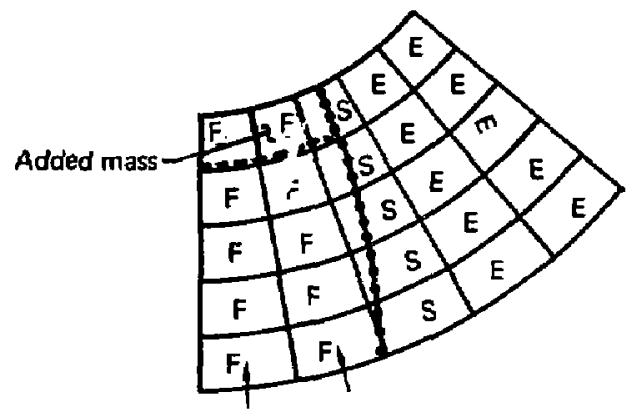

(b) Artificial mass creation due to finite cell size and flagging scheme.

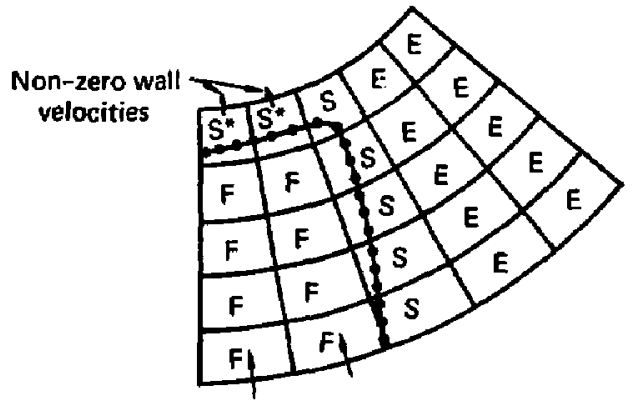

(c) Special surface cells allow fluid to enter cells near wall.

Figure 01 . Special surface cells prevent artificial mass creation near walls. 
SURFACE MARKER CHAINS

Replacing marker particles in the fluid body with a chain of particles at the surface is a significant improvement in MAC methodology $(D 6,07)$. The surface marker chain reduces the number of particles and computer storage required and provides more accurate location of the surface. With the surface precisely located within a cell, the pressure can be extrapolated or interpolated from the nearest full neighbor to the exact surface location instead of the cell center. The essential featires of the surface marker chain are: 1) sequential particle numbering, 2) maintenance of uniform particle spacing, and 3) attachment of chain ends to walls.

When particles move, they tend to bunch up or thin out. As a result, we must add or delete particles during each computational cycle to keep uniform spacing and accurately mark the surface. After editing particle spacings the chain ends are attached by moving the first and last particles radially to the walls. This prevents chain ends from drifting away from the walls.

When a surface wave breaks an interior cell may jump from empty to full in a single cycle as illustrated in Fig. D2. Special surface cells handle this situation near watis but do nothing for interior cells. Motion of the type $i$ llustrated results in a folded and tangled surface chain with some particles that may not lie on the surface. To keep the chain from tangling and determine when chain splitting is necessary, particles in full cells are removed each computational cycle. After particles are removed the gap distance is checked to determine if the chain should be split. If the gap exceeds 2.5 times tiie local cell 


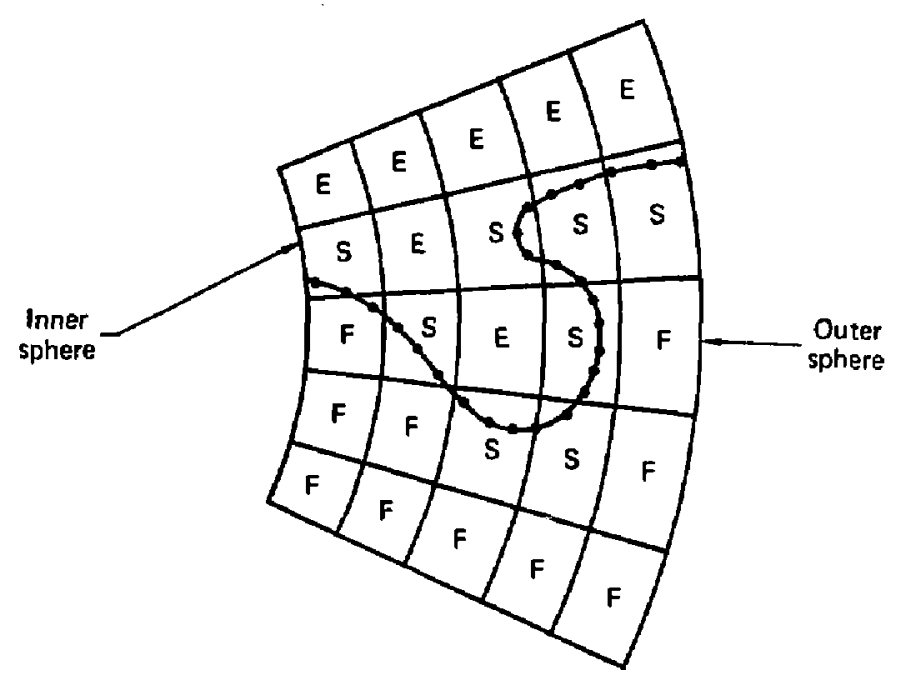

(a). Breaking wave about to collapse on a single empty cell.

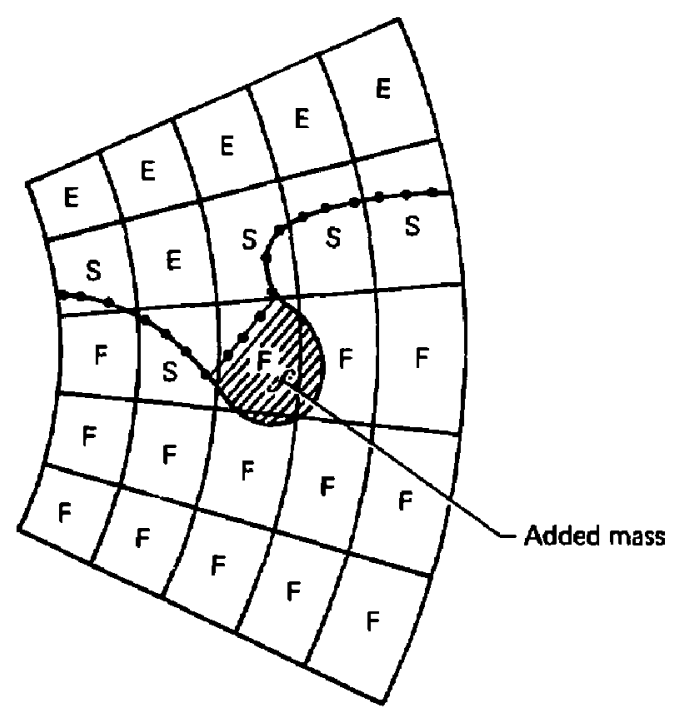

(b). Added mass due to renoval of particies in full cells.

Figure D2. Cell resolution and chain straightening cause artificial mass creation. 
diagonal and is near a boundary, the chain is split and the new end points are attached to the wall. If the chain is not split, particles are replaced in the full cell along a straight line segment. The effect of the straight line replacement is straightening of the surface chain. He will see the advantage of straightening the surface chain later when we attempt to find the surface location in each surface cell.

As a result of chain straightening and interior cells jumping from empty to full, the total system fluid mass increases as illustrated by the shaded area in Fig. D2. A small increase in mass occurs every time we straighten a segment of surface chain and when a wave breaks. Repeated mass errors of this type add up to the point of causing significant error in the total system mass. We have not found a feasible way to prevent mass creation when a surface breaks. This error is incurred due to the finite size of the computational cells. In addition, if we do not destroy particles in full ce?ls each cycle, the surface chain becomes tangled and causes problems for surface pressure interpolation. A solution to the added mass problem is to correct the surface location periodically. We will discuss a method of doing this in the section on surface adjustment.

He have discussed what happens when the surface folds over and creates a bubble that is smaller than the local cell size. Another situation we must deal with is a bubble larger than the local cell size as illustrated in Fig. 03. In this situation, the chain is split to mark different sections of the curface. The two types of surface chains (regular and bubble) are shown in part $d$ of Fig. 03. Both chains are treated identically with the exception of the chain ends. A regular 


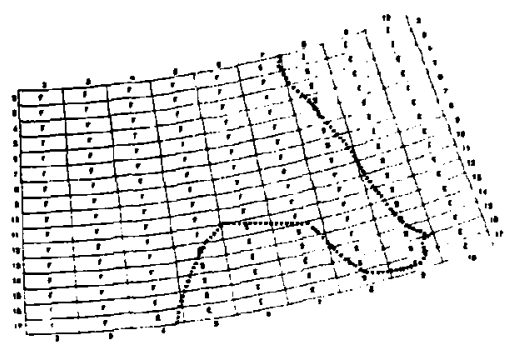

(a) $\mathrm{t}=.150$ seconds

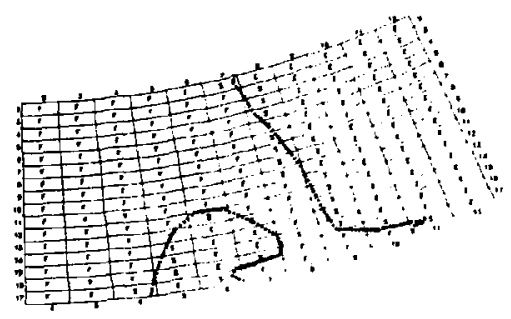

(c) $t=.162$ seconds

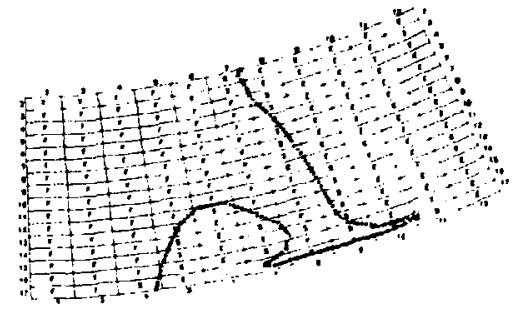

(b) $t=.159$ seconds

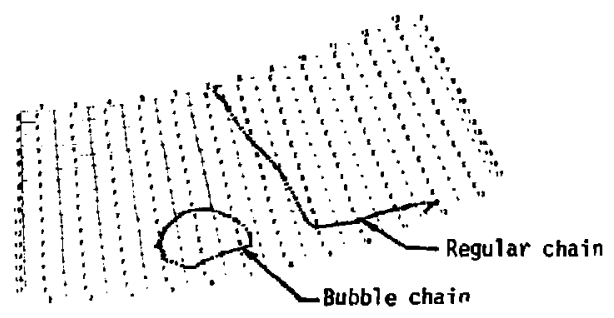

(d) $t=.174$ seconds

Figure 03. Sequence of inlet jet forming a bubble and surface marker chain splitting. 
chain is attached at both ends to houndaries and a bubble chain is attached to itself head to tait. When the bubble size shrinks to the order of the local cell, all surface cells are flagged full and the bubble chain is destroyed. Once again the unavoidable jump of a cell flag from empty to full will occur.

\section{FREE SURFACE VELOCITY COWOITIONS}

The first step in calculating velocities at a free surface is to insure that mass is conserved in all surface cells. This is done by using equation 13 from Section IV to calculate velocities on surface cell faces adjacent to empty cells. The configuration of a surface cell with a single empty neighbor appears commonly and is shown in Fig. 04. The velocity component on the top face is calculated to make the cell divergence zero. The position of this velocity is indicated by a dot and the arrows on the sides of the surface cell indicate velocities calculated from the momentum equations. The dashed arrows indicate borrowed velocities. The calculation of these borrowed velocities will be discussed in a following section on surface stress conditions. More complicated configurations exist when there is more than one empty neighbor and more than one unknown velocity.

The possible arrangements of empty cells abcut a single surface ceil and a numerical scheme to determine the configuration were explained in detail by Amsden et. a1. (D1). The scheme involves an integer sum varying from 1 to 15 based on the arrangement of eimpty cells about a surface cel1. Figure 05 shows the fifteen arrangments and the assigned numbers. The dot indicates the zero divergence velocity and other 


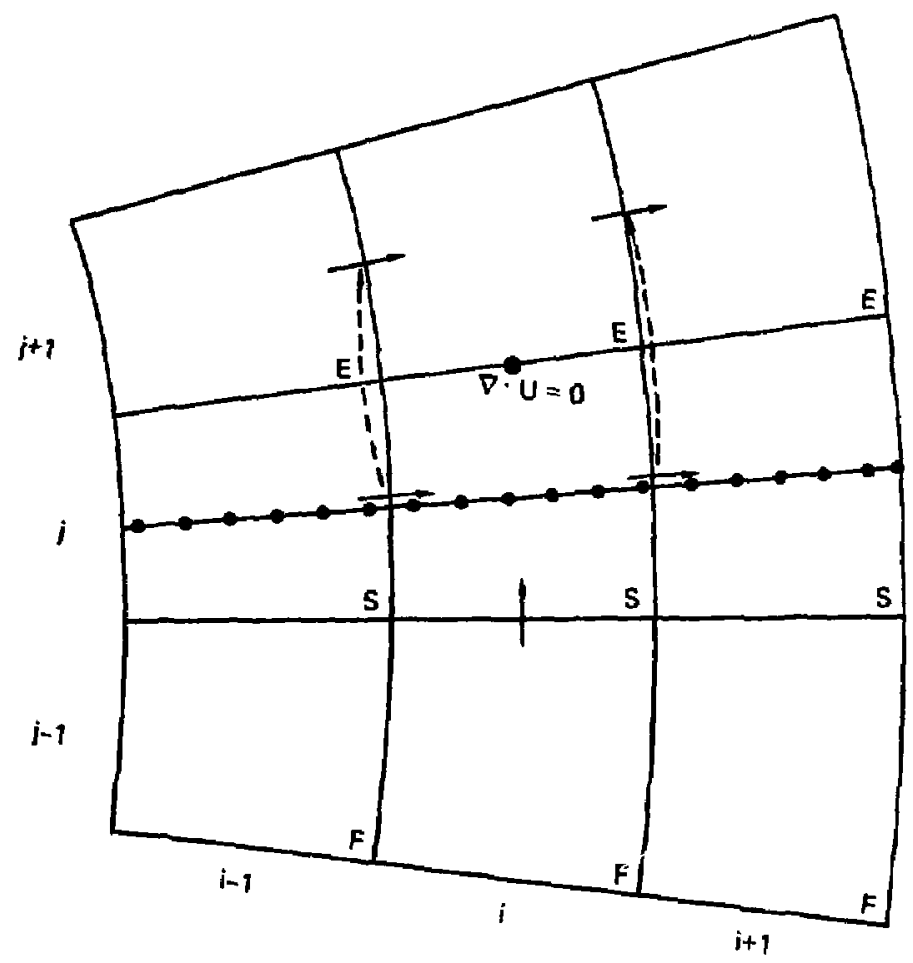

Figure 04. Surface velocity conditions for surface cell with a single empty neighbor. 


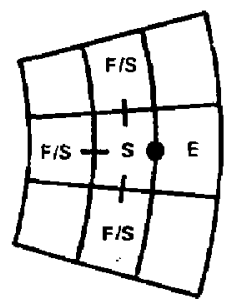

1

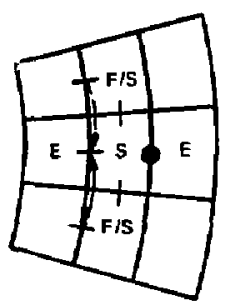

5

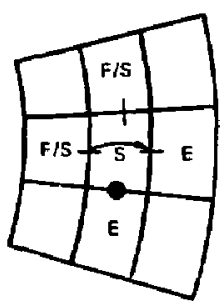

9

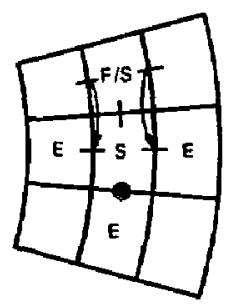

13

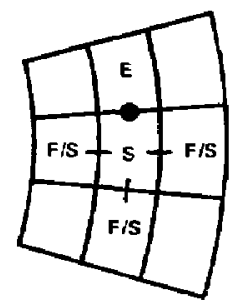

2

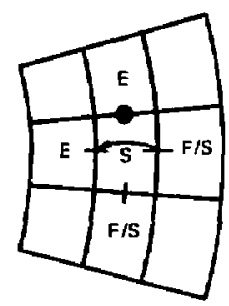

6

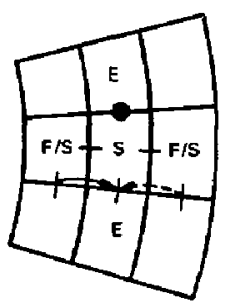

10

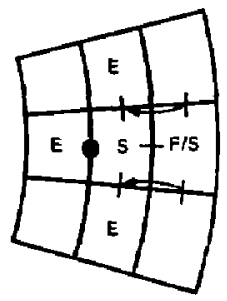

14

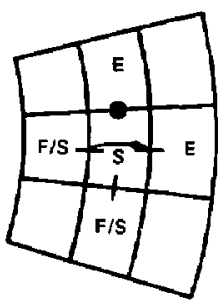

3

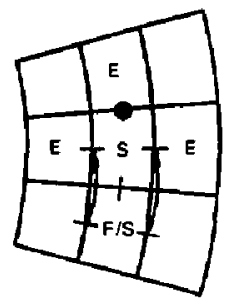

7

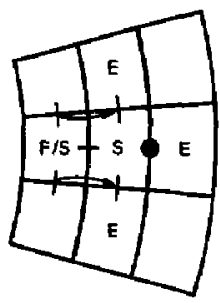

11

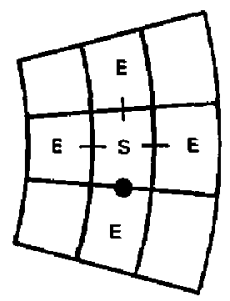

15

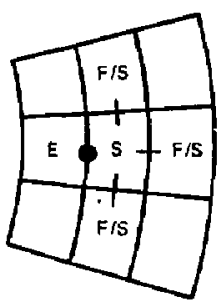

4

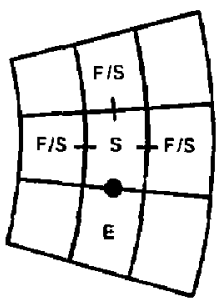

8

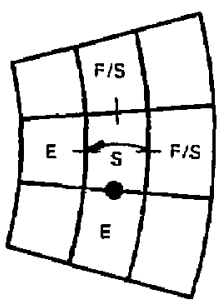

12

Figure 05. Surface-empty cell arrangements and velocity treatment for each. 
velocity components are borrowed from neighboring full cells as indicated by the curved arrows. In each case the borrowed velocity is adjusted to maintain equal mass flow. Our treatment differs from early methods, primarily because each cell face has a different area. When there is more than one unknown velocity the treatment is not rigorous (07).

Nonzero wall velocities for special surface cells are calculated in this portion of the calculation. Figure 06 shows a typical configuration of surface and special surface cells and the positions of the zero divergence velocities.

\section{SURFACE ADJUSTMENT}

Added mass caused by cell resolution and chain straightening must be accounted for. This is accomplished by periodically adjusting the surface location. The surface position is adjusted by setting a nonzero divergence in all surface cells for a portion of the calculation that affects only the particle movement. During each computational cycle, theoretical fluid volume is compared to the volume of all full cells plus surface cells. The theoretical volume should be between the full cell volume and the combined volume of full cells plus surface cells. If the thearetical fluid volume is outside these limits, then the calculated volume is adjusted. Excess volume is calculated from the following equation:

$$
v_{e x}=\left(v_{t}+v_{s} / 2-v_{t h}\right)
$$




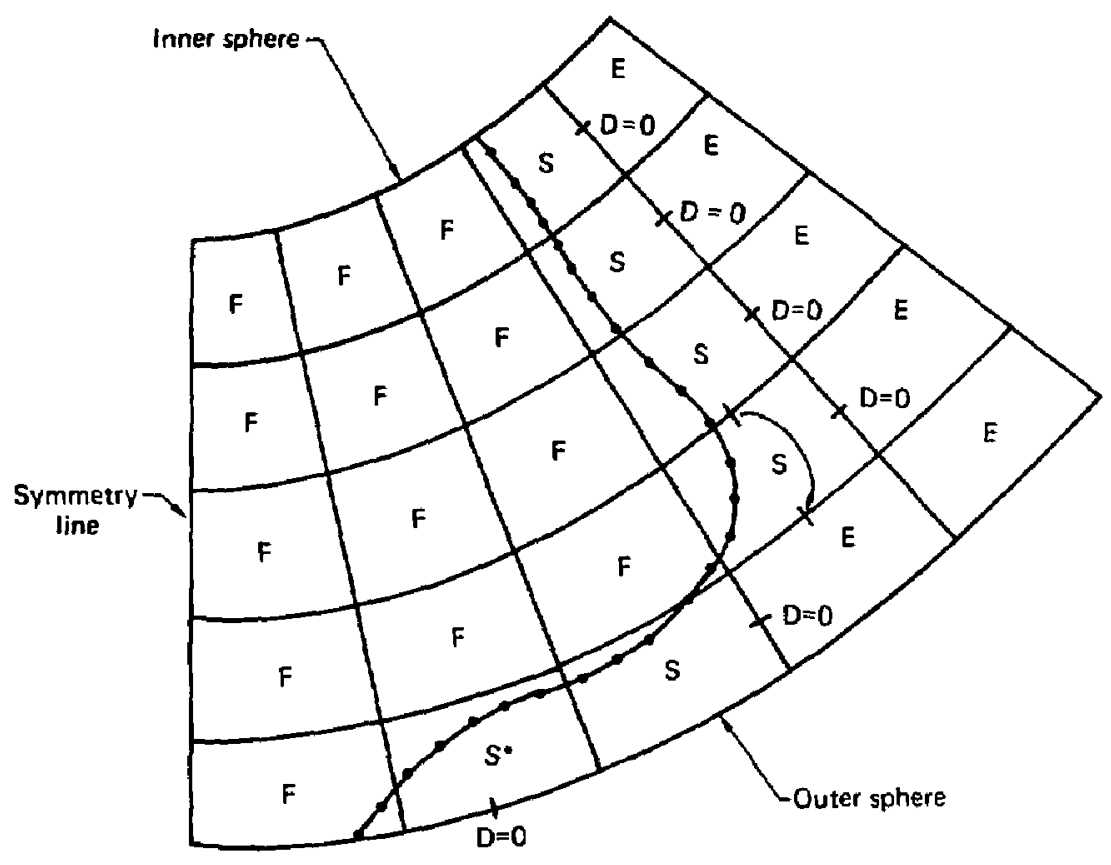

Figure DS. Typical cell configuration and velocity treatment for regular and special surface cells. 
In the above equation, $V_{f}$ is the summed volume of all full cells, $V_{s}$ is the summed volume of all surface cells, $V_{\text {th }}$ is the thearetical volume and $v_{e x}$ is the excess volume. A nonzero divergence for each surface cell is calculated from the oxpression

$$
D_{e x}=-\frac{V_{e x}}{v_{s} \Delta t}
$$

The condition for surface cell velocities adjacent to empty sells during the adjustment portion of the cycle is

$$
\vec{\nabla} \cdot \vec{v}=D_{\text {ex }} \text {. }
$$

in place of $\vec{\nabla} \cdot \vec{U}=0$. The nonzero surface cell divergence is used only for particle movement and is set back to zero before the cycle is continued. The effect is an adjustment in surface velocities by the proper amount to correct in a single time step for an existing volume error. The adjusted surface velocities cause a small increment to be added to or subtracted from the location of the surface chain and force the calculated fluid volume to be approximately equal to the theoretical volume.

The approximate method used to calculate excess volume is accurate to within the order of the size of surface cells and provides a simple method of volume adjustment. Volume adjustment is required only periodically during the computation. The more irregularity in the surface shape, the more. of ten the correction is required. 


\section{SURFACE STRESS CONDITIONS}

For low Reynolds number flows, tangential and normal stress conditions can be applied at the surface to calculate pressure in a surface cell and velocities just outside the surface. For this problem we use the inviscid surface stress condition as suggested by Nichols and Hirt (D4). To approximate the inviscid free surface, we set $P=0$ at the surface and obtain velocities outside the surface by reflecting mass flow from below the surface. For the case shown in Fig. D4, the outside velocity is calculated according to

$$
u_{i+1 / 2, j+1}^{n}=u_{j+1 / 2, j}^{n} \frac{\sin \theta_{j} \Delta \theta_{j}}{\sin \theta_{j+1} \Delta \theta_{j+1}} .
$$

The inviscid form of the normal stress condition is applied by setting pressure equal to zero at the surface. Originally in MAC, the surface was assumed to be at the center of the surface celi and the surface pressure was applied there. With the addition of the surface marker chain, it became possible to set the surface pressure exactly at the free surface location.

\section{SURFACE PRESSURE INTERPOLATION}

The surface pressure is applied at the exact surface location by interpolating or extrapolating pressure along the line between surface and adjacent full cell centers. To accomplish the interpolation, the location where the surface crosses this line must be known. In Fig. D7 we show a typical surface configuration and the distance needed for interpolating or extrapolating zero pressure to the surface. 


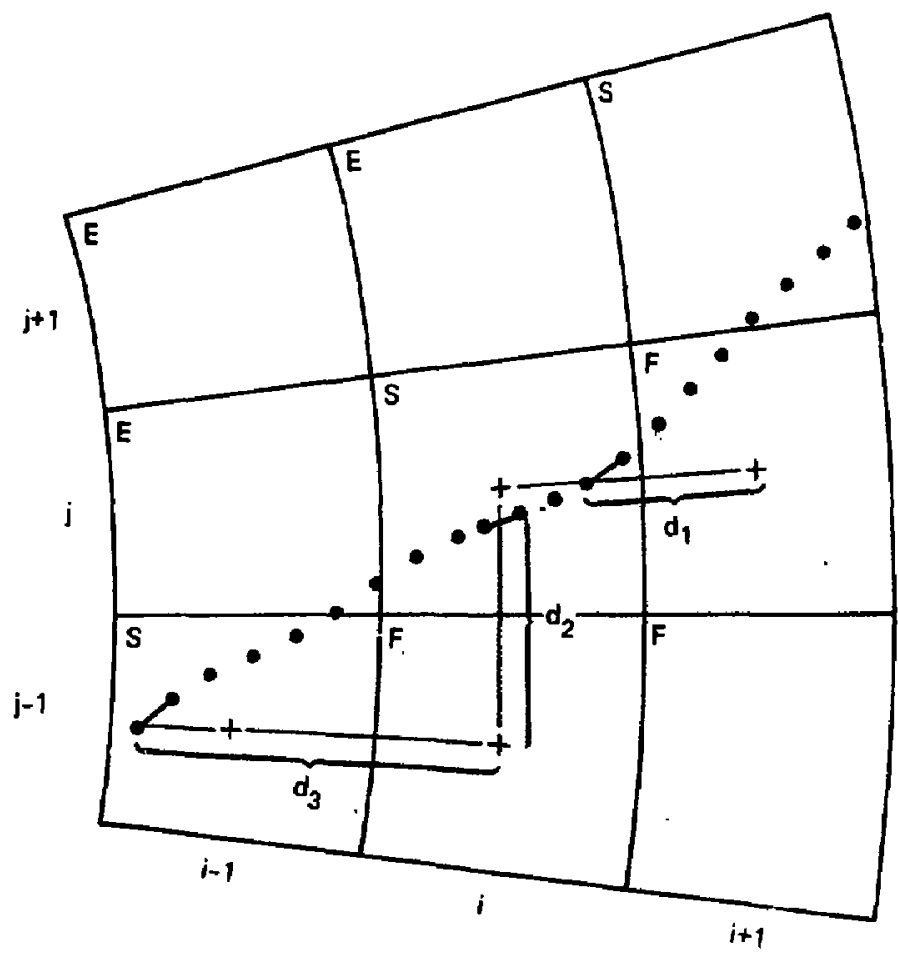

Figure 07. Surface pressure interpotation distances. 
To Tocate the intersection point of the surface chain and the line between cell centers, we march along the chain and note where the $r$ and/or $\theta$ coordinate of the neighboring full cell is crossed. There are two such points for cell $(i, j)$ shown in Fig. D7. Finding these points can be difficult if the surface chain is irregular. If the chain is folded on itself and crosses the line several times, it may be impossible to locate the correct point. Chain straightening plays a very important role by keeping the surface chain regular and single valued inside a cell.

The interpolation neighbor for each surface cell is chosen based on the minimum distance in cell units from the cell center to the fluid surface. The expressions used to determine the intepolation neighbor for cell $(i, j)$ in Fig. 07 are

$$
\left|1-\frac{d_{1}}{r_{i+1}-r_{j}}\right| \quad \text { and } \quad\left|1-\frac{d_{2}}{r_{i}\left(\theta_{j}-\theta_{j-1}\right)}\right|
$$

The minimum of these quantities dictates the full celt interpolation reighbor for surface cell $(i, j)$. The interpolation factor $n$ is then calculated and according to

$$
\eta=\frac{r_{i+1}-r_{i}}{d_{1}} \quad \text { or } \quad \eta=\frac{r_{i}\left(\theta_{j}-\theta_{j-1}\right)}{d_{2}}
$$

depending on which full cell is chosen as the interpolation neighbor. The pressure in surface cell $(i, j)$ is calculated by linear interpolation according to the equation 


$$
P_{a}=(1-\eta) P_{1}
$$

where $P_{S}$ is the surface cell pressure and $P_{f}$ is the pressure of the full cell interpolation neighbor. Equation D-5 is used to update the surface cell pressure during the iteration phase of the calculation. On each sweep through the mesh, new surface cell pressures are calculated from updated pressures in the full neighbors. In addition, velocities on cell faces between surface and full cells are updated to reflect the new pressure gradients.

There are limits that must be placed on the interpolation parameter $\eta$. By placing the lower limit at 0.667, we effectively limit the maximum distance d in Fig. 07 to 1.5 cell widths. This limit is reached when the surface intersects the line between cell centers an the empty side of the surface cell. If the intersection point lies in the full ce 17, then $n$ is greater than 2.0 and an instability in the pressure-velocity iteration will occur (D4). In this case, a change in full cell pressure will cause a greater change in surface cell pressure and on the next iteration the full cell pressure will change by a larger amount in the opposite direction to account for the change in surface cell pressure. This process rontinues and the pressures oscillate and diverge. To prevent iterative divergence a relaxation parameter $\omega_{f}$ for use in equation 16 is calculated that limits changes in the full cell pressure depending on the value of $n$.

To derive $w_{f}$, we begin by rewriting equations 14 (Section IV) in the form 


$$
u_{i+1 / 2, j}^{n+1}=\hat{u}_{i+1 / 2, j}^{n+1}+\frac{\Delta t}{\rho\left(r_{i+1}-r_{i}\right)}\left(P_{i+1, j}^{n}-P_{i, j}^{n}\right)
$$

$$
v_{i, j+1 / 2}^{n+1}=\hat{v}_{j, j+1 / 2}^{n+k}+\frac{\Delta t}{\rho \mathbf{r}_{j}\left(\theta_{j+1}-\theta_{j}\right)}\left(P_{i+1, j}^{n}-P_{i, j}^{n}\right),
$$

etc. for all cell velocity components. We have dropped the pressure adjustment term $\Delta \mathrm{P}^{\mathrm{I}}$ and pulled the pressure derivative term outside the tilde velocities. The new velocity terms $i_{i+1 / 2, j}^{n+1}$ and $\hat{v}_{i, j+1 / 2}^{n+1}$ etc. are equivalent to the right hand sides of equations 10 and 1.1 without the pressure derivative. Next, the cell velocity components from the above equations are substituted into equation 12 (Section IV) and rearranged into the following expression:

$$
\begin{aligned}
& \left.\qquad \bar{P}_{i, j}^{1+1}=\frac{1}{\beta_{i+j} r_{i}^{2}}\right\} \frac{1}{\Delta r_{i}}\left[\frac{r_{i+1 / 2}^{2}}{\Delta r_{i+1 / 2}} P_{i+1, j}^{n}+\frac{r_{i-1 / 2}^{2}}{\Delta r_{i-1 / 2}} P_{i-1, j}^{n}\right] \\
& \left.+\frac{1}{s i n \theta_{j} \Delta \theta_{j}}\left[\frac{s i n \theta_{j+1 / 2}}{\Delta \theta_{j+1 / 2}} P_{i, j+1}^{n}+\frac{s i n \theta_{j-1 / 2}}{\Delta \theta_{j-1 / 2}} P_{i, j-1}^{n}\right]-\frac{s_{i, j} r_{i}^{2}}{\Delta t}\right\} \\
& \text { Grid spacing terms such as } \Delta r_{i} \text { and } \Delta \theta_{j} \text { were previously def ined and } B_{i, j} \text { is } \\
& \text { given by equation } 17 \text { in Section IV. The source term } S_{i, j} \text { is defined by } \\
& \text { the equation }
\end{aligned}
$$




$$
\begin{gathered}
s_{i, j}=\frac{1}{r_{i}^{2}}\left[\frac{r_{i+1 / 2}^{2} \hat{u}_{i+1 / 2, j}^{n}-r_{i-1 / 2}^{2} \hat{u}_{i-1 / 2, j}^{n}}{r_{j+1 / 2}-r_{i-1 / 2}}\right] \\
+\frac{1}{r_{i} \sin \theta_{j}}\left[\frac{\sin r_{j+1 / 2} \hat{v}_{j, j+1 / 2}^{n}-\sin \theta_{j-1 / 2} \hat{v}_{i, j-1 / 2}^{n}}{\theta_{j+1 / 2}-\theta_{j-1 / 2}}\right] .
\end{gathered}
$$

The pressure given by equation $D-7$ is the Gauss-Siedel iterative vaiue for pressure at the $I+1$ iteration and is denoted as $\mathrm{P}_{\mathbf{i}, j}^{\mathrm{I}+1}$. At this point, we drop the time level superscript $n$, and indicate iteration level by superscript I. For successive-over-relaxation (SOR), the Gauss-Siede 1 value $(D B)$ is extrapolated using the relaxation parameter $\omega_{0}$ according to

$$
P_{i, j}^{1+1}=\left(1-\omega_{0}\right) P_{i, j}^{1}+\omega_{0} \overline{P_{i}}+i_{j}^{+}
$$

In using this equation, the mesh is swept and pressures are updated continuously resulting in trailing cells $(i-1)$ and $(j-1)$ at iteration level $I+I$ and advance cells $(i+1)$ and $(j+1)$ at iteration leve 1 .

The purpose of this derivation is to find an equivalent relaxation parameter for a full cell interpotation neighbor. We will do this for the case of a fult cell at $(i, j)$ and a surface cell at $(i, j+1)$. In equation $0-7, P_{i, j+1}^{n}$ is replaced by the interpolated va? ie at the new iteration level

$$
P_{i, j+1}^{n} \rightarrow\left(1-\eta_{i, j+1}\right) P_{i, j}^{l+1}
$$

The result is substituted into equation $0-9$ to yield 


$$
\begin{aligned}
& P_{i, j}^{l+1}=\left(1-\omega_{0}\right) P_{i, j}^{l} \\
& +\frac{1}{\sin \theta_{j} \Delta \theta_{j}}\left[\frac{\sin \theta_{j+1 / 2}}{\Delta \theta_{j+1 / 2}}\left(1-\pi_{i, j+1}\right) P_{i, j}^{l+1}+\frac{\sin \theta_{j-1 / 2}}{\Delta \theta_{j-1 / 2}} P_{i, j-1}^{1+1}\right] \\
& +\frac{\omega_{0}}{\beta_{1, j} r_{i}^{2}}\left\{\frac{1}{\Delta r_{1}}\left[\frac{r_{i+1 / 2}^{2}}{\Delta r_{i+1 / 2}} P_{i+1,1}+\frac{r_{i-1 / 2}^{2}}{\Delta r_{i-1 / 2}} P_{i-1,1}^{l}\right]\right. \\
& \left.-\frac{s_{1, j} r_{i}^{2}}{\Delta t}\right\}
\end{aligned}
$$

Next, we bring $P_{i, j}^{I+I}$ outside the brackets and rearrange terms to recover the Ganss-Siede? value as given by the right hand side of equation $0-7$. - sition is then cast inta a form similar to equation D-9.

$$
P_{i, j}^{l+1}=\left[1-\frac{\omega_{0}}{1-\omega_{0} \gamma_{i, j+1}}\right] P_{i, j}^{1}+-\frac{\omega_{0}}{\omega_{0} \gamma_{i, j+1}} \bar{P}_{i, j}^{l+1}
$$

where:

$$
\gamma_{1, j+1}=\frac{1 \rightarrow \eta_{j, j+1}}{\beta_{1, j} r_{i}^{2}}\left\{\frac{\sin \theta_{j+1} / 2}{\sin \theta_{j} \Delta \theta_{j} \Delta \theta_{j+1 / 2}}\right\}
$$

By comparing the form of equation D-1c ith equation D-9 we see that the new relaxation parameter for the full interpolation cell has the form

$$
\omega_{1}=\frac{\omega_{0}}{1-\omega_{0} \gamma_{i, 1+1}}
$$


We also note in this equation that $\omega_{f}=\omega_{0}$ for $n=1$. For a full cell at $(i, j)$ and surface cell at $(i, j+1), \gamma$ is given by equation $D-13$. The subscripts on $r$ and $n$ indicate the location of the surface cell in relation to full cell $(i, j)$. By repeating the above steps for the other possible interpolation neighbors we arrive at the following expressicas. For full cell at $(i, j)$, surface cell at $(i, j-1)$ :

$$
\gamma_{i, j-1}=\frac{1-\eta_{i, j-1}}{\beta_{i, j} T_{i}^{2}}\left\{\frac{\sin \theta_{j-1 / 2}}{\sin \theta_{j} \Delta \theta_{j} \Delta \theta_{j-1 / 2}}\right\} .
$$

For full cell at $(i, j)$, surface cell at $(i+1, j)$ :

$$
\gamma_{i+1, j}=\frac{1 \eta_{i+1, j}}{\beta_{i, j} r_{i}^{2}}\left\{\frac{r_{i+1 / 2}^{2}}{\Delta r_{i} \Delta r_{i+1 / 2}}\right\} .
$$

Full cell at $(i, j)$, surface cell at $(i-1, j)$ :

$$
\gamma_{i-1, j}=\frac{1-\eta_{i}-1, j}{\beta_{i, j} r_{i}^{2}}\left\{\frac{r_{i-1 / 2}^{2}}{\Delta r_{i} \Delta r_{i-1 / 2}}\right\} \text {. }
$$

The relaxation parameter given by equation $0-14$ will maintain a stable iteration for all values of $\eta$ when a single full cell pressure is interpolated or extrapolited to a single surface cell.

In some situations, it is possible for a single full cell to act as an interpolation neighbor for more than one surface cell. This is the

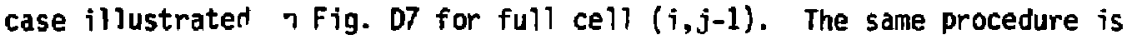
followed to derive $\omega_{f}$ for a double interpolation as for a single interpolation. This time, we replace two interpolated values into equation 0-7 for the appropriate surface cell pressures. The resulting expression 
for $w_{f}$ is identical to equation D-14 with differences appearing in the form of $\gamma$. For interpolation by a single full cell to two surface cells, the expression for $\gamma$ turns out to be the sum of $\gamma$ for the individual single interpolations. For example

$$
\gamma_{(i+1, j) \cdot(i, j+1)}=\gamma_{i+1, j}+\gamma_{i, j+1}
$$

Double interpolutions are far less common than single and are avoided if single interpolation can be done. Iterative convergence is slowed down somewhat when $n>2$, especially if a double interpolation is being cone. 


\section{REFFERENCES FOR APPENDIX D}

D1. A. A. Amsden, F. H. Harlow, The SHAC Methad: A Numer ical Technique for Calculating Incompressible Fluid Flows, Los Alamos Scient if ic Laboratory Report 'LA-4370 (1970).

D2. C. W. Hirt and J. P. Shannon, "Free Surface Stress Conditions for Incompressible-Flow Calculations," J. Comp. Phys., Vol 2, (1968), p. 403.

D3. R. K.-C. Chan and R. L. Street, "A Computer Study of Finite-Amplitude Water Waves," J. Comp. Phys., Vol, 6, (1970), p. 68 .

D4. B. D. Nichols and C. W. Hirt, "Improved Free Surface Boundary Condition for Numerical Incompressible-Flow Calculations," Comp. Phys., Vol. 8, (1971), p. 434.

D5. C. W. Hirt, B. D. Nichols, and N. C. Romero SOLA -- A Numerical Solution Algorithm for Transient Fluid Flows, Los Alamos

Scientific Laboratory Report LA-5852 (1975).

D6. B. D. Nichols: "Recent Extensions to the Marker-and-Cell Method for Incompressible Fluid Flows," Proceedings Second International Conference on Mumerical Methods in Fluid Dynamics (Springer-Yerlag, Berkeley, California, 1970), p. 371.

D7. A. A. Amsden, Numerical Calcurations of Surface Haves: A Modified ZUNI Code with Surface Particles and Partial Cells, Los Alamos Scientific Laboratory Report LA-5146 (1973).

D8. H. F. Ames, Numerical Methods for Partial Differential Equations (Barnes and Noble Publishing, New York, 1969). 


\section{APPENDIX E}

\section{THE COMPUTER CODE SAFFA}

The computer code used for these calculations is named SAFFA, for Spherical Annu?us Fluid-Flow Algorithm. A flow chart illustrating the code logic is shown in Fig. El.

The logic consists of a main time roop composed of three phases. The first phase deals with the computation of fluid velocities, pressures, $\cdots$ temperatures. In the second phase the free surface and marker particles are handled and in the third phase, prints, plots, and restart dumps are made.

Surface and houndary cell velocities are set before particle mavement so that particles maybe moved based on a completely updated velocity field. After particles are moved, cell flags are changed and surface and boundary cell velocities are set again to reflect changes in cell flags.

The Fortran source deck contains approximately 4000 cards including comments, printing, plotting, and input subroutines. An average calculation of an annulus fill requires about 1 hour on the CDC 7600. The code is still being developed and has not been streamined for production work. 


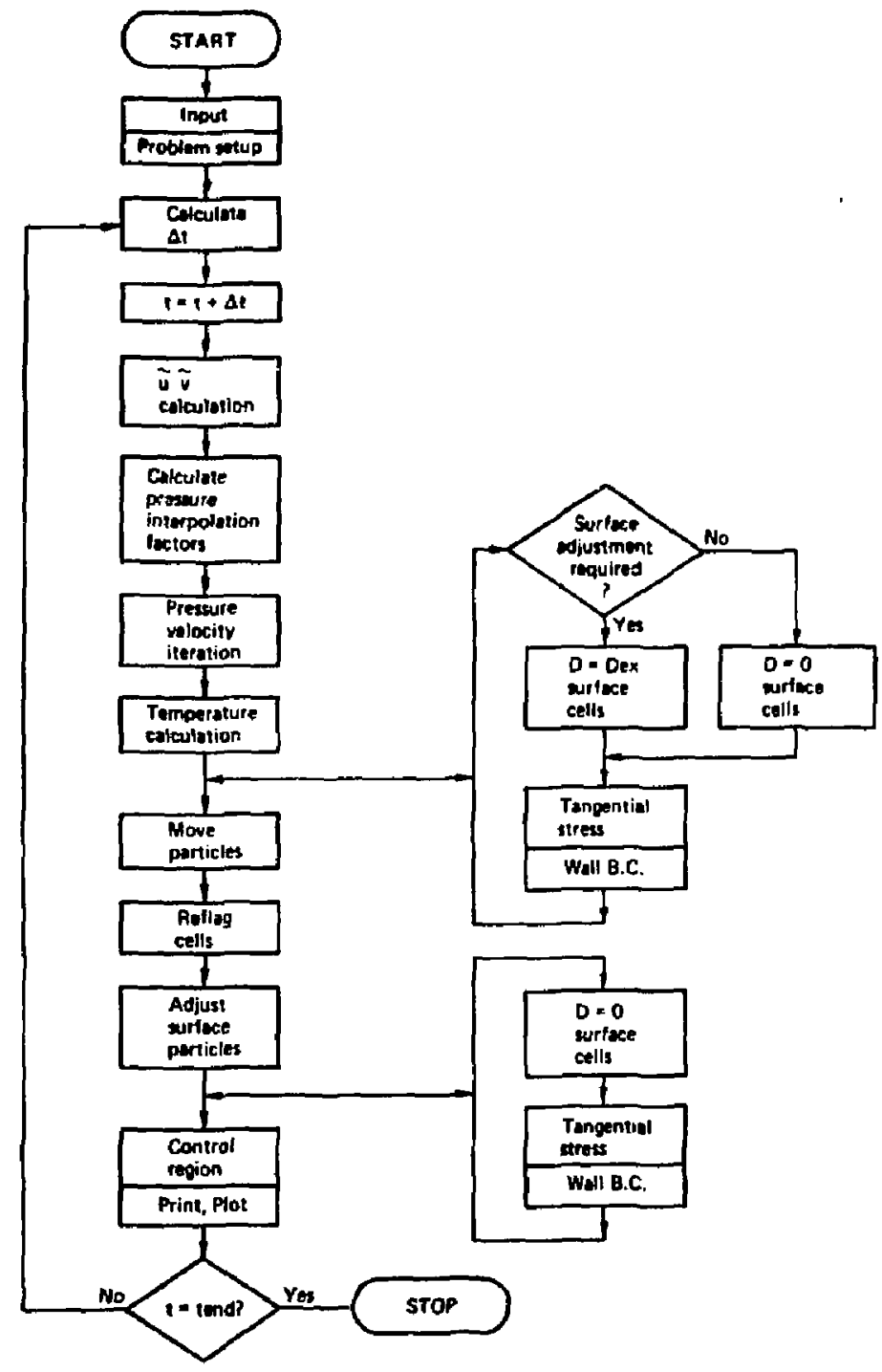

Figure El. Flow diagram for SAFFA computer code. 


\section{APPENDIX F}

NOMENCLATURE

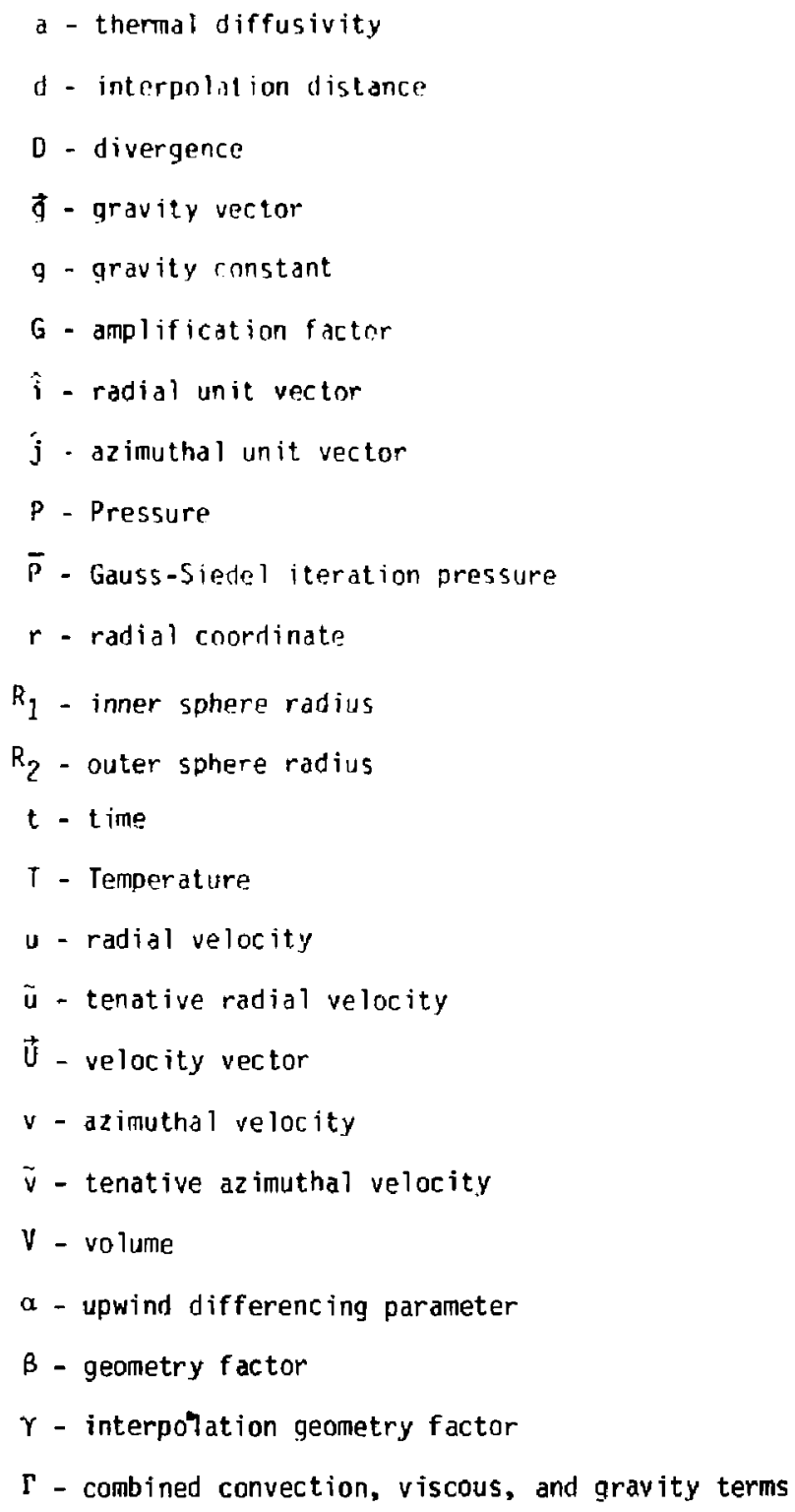


a - partial derivative

$A$ - interval of change

n - interpolation parameter

0 - az imuthal coordinate

$\Lambda$ - vorticity

$v$ - kinematic viscosity

P - density

$T$ - shear stress

\$ - longitudinal coordinate

- phase angle

$\psi$ - amplitude function

$\omega$ - over-relaxation parameter

Vector Operators

$\forall$ - spherical gradient

$\nabla^{2}$ - Laplacian

Subscripts

$$
\begin{aligned}
& \text { ex - excess } \\
& f \text { - full } \\
& \text { in - inside } \\
& i \text { - radial cell index } \\
& j \text { - azimuthal cell index } \\
& \text { in - inside } \\
& \text { out - outside } \\
& r \text { - radial direction } \\
& s \text { - surface } \\
& \text { th - theoretical } \\
& \theta \text { - azimuthal direction }
\end{aligned}
$$


1 - iteration number

$N$. t ime step number

- special surface cell 